\title{
Ligand-Programmed Consecutive Symmetry Break(s) in Nanoparticle Based Materials Showing Emergent Phenomena: Transitioning from Sixfold to Threefold Symmetry in Anisotropic ZnO Colloids
}

\author{
Sebastian Theiss, Michael Voggel, Henning Kuper, Martin Hoermann, Ulrich Krings, \\ Peter Baum, Joerg August Becker, Valentin Wittmann, and Sebastian Polarz*
}

The central promise of nanoparticle-based materials is that cooperative properties may emerge, when individual quantum dots are positioned on a periodic lattice. Yet, there are only a few papers in the literature reporting such effects. Nevertheless, it is clear that the symmetry of the superlattice is decisive for the desired emergent phenomena. An interesting question is, how the symmetry of the initial monodisperse nanoparticles affects the structure of the colloidal crystal during self-assembly processes. For instance, particles with a hexagonal cross-section demonstrate self-organization, which is very similar to spherical colloids. Likewise, one would also expect that trigonal nanoparticles behave similarly. Unfortunately, it is very hard to obtain monodisperse semiconductor colloids with a trigonal shape, because this requires a symmetry break during morphogenesis of the nanocrystal. While such a symmetry break is known in the literature for structures attached to a solid substrate, herein, colloidal synthesis of trigonal $\mathrm{ZnO}$ nanorods is successfully demonstrated, and the mechanism is elucidated via experimental and theoretical methods. 2D-superlattices formed by such particles with trigonal cross-section are compared to hexagonal analogues. It is found that there are distinct differences, which result in important differences in properties such as the formation of voids and also in optical properties.

\section{Introduction}

Already in the beginning of research on semiconductor quantum dots, the pioneers in the field envisioned that nanoparticles will become key constituents in future technologies..$^{[1-3]}$ However, it took a while until we saw semiconductor nanoparticles (SNs) in products such as displays/light emitting diodes (LEDs). A reason for the delay is, gaining profound control over size, shape, composition, sizedistribution and surface chemistry had to be learned first. There are two distinct variants for using SNs in functional materials. The dominant approach is to embed isolated SNs in a matrix of a second material, e.g., a polymer, respectively to prepare a blend. Because significant progress in the making of monodisperse SN dispersions by colloidal synthesis has been achieved, ${ }^{[4,5]}$ superstructures formed by assembly of such nanoparticle building blocks became more and more interesting. ${ }^{[6,7]}$

Particle-based material can be categorized into two categories, randomly- and periodically ordered architectures. Examples for the first category are photonic glasses $^{[8]}$ or nanoparticle-based aerogels, ${ }^{[9-11]}$ which are characterized by the absence of long-range order. The motivation for periodic nanoparticle-based materials lies within the amplification of existing- or even the expectation of new properties to appear when nanoparticles are arranged within close ordered
S. Theiss, Prof. S. Polarz

Leibniz-University of Hannover

Institute of Inorganic Chemistry

Callinstrasse 9, 30167 Hannover, Germany

E-mail: sebastian.polarz@aca.uni-hannover.de

The ORCID identification number(s) for the author(s) of this article can be found under https://doi.org/10.1002/adfm.202009104.

(C) 2020 The Authors. Advanced Functional Materials published by Wiley-VCH GmbH. This is an open access article under the terms of the Creative Commons Attribution-NonCommercial-NoDerivs License, which permits use and distribution in any medium, provided the original work is properly cited, the use is non-commercial and no modifications or adaptations are made.

\author{
S. Theiss, M. Voggel, M. Hoermann, Prof. P. Baum, \\ Prof. V. Witmann, Prof. S. Polarz \\ University of Konstanz \\ Universitaetsstrasse 10, 78457 Konstanz, Germany \\ H. Kuper, Prof. J. A. Becker \\ Leibniz-University of Hannover \\ Institute of Physical Chemistry \\ Callinstrasse 3A, 30167 Hannover, Germany \\ Dr. U. Krings \\ Leibniz-University of Hannover \\ Institute of Food Chemistry \\ Callinstrasse 3-9, 30167 Hannover, Germany
}

DOI: 10.1002/adfm.202009104 
proximity. Thereby, the probability rises that electronic-states of individual nanoparticles overlap with their nearest neighbors and symmetry equivalents to form a superordinate electronicstate of the superstructure, which may result in the appearance of cooperative effects. Examples for periodic nanoparticle based materials involve thin-films, ${ }^{[12]}$ smectic phases, ${ }^{[13]}$ interlocking networks, ${ }^{[14]}$ chiral helices, ${ }^{[15]}$ cylinders ${ }^{[16]}$ or mesocrystals. ${ }^{[17]}$ Similar to atomic crystals, the symmetry of the lattice is a deciding factor for such emergent properties.

There is one important difference, if one wants to compare SNs to atoms and their periodic assembly into crystals. Atoms are spherical, while SNs may not. Shape anisotropic nanoparticles offer far more possibilities of assembly and superstructure formation. ${ }^{[18]}$ They can appear in various shapes like nanorods or nanoneedles (quasi 1D), nanoplates or sheets (quasi 2D), tetra-, octa- or multipods (3D) or other morphologies. Hence, more attention needs to be given to a profound "nano-metrology" and nomenclature as described in the literature. ${ }^{[19,20]}$

A key in achieving shape control is the use of ligands, respectively capping agents, during the colloidal synthesis methods. These ligands are often small organic molecules with amphiphilic character like phosphonic-acids, ${ }^{[21-23]}$ amines, ${ }^{[24-26]}$ thiols, ${ }^{[27,28]}$ or carboxylic-acids ${ }^{[29-32]}$ that exhibit a certain affinity towards the surface of the nanoparticles. Intrinsically different growth rates represent another factor which influences the shape of SNs. For instance, for polar, non-centrosymmetric crystal systems (e.g., wurtzite, space group P63mc), an anisotropic growth in $c$-direction can occur naturally. Among others $(\mathrm{ZnS}, \mathrm{GaN}, \mathrm{CdTe})$ zinc oxide $(\mathrm{ZnO})$ is one prominent member of this class. $\mathrm{ZnO}$ is a multifunctional, wide-gap semiconductor and the reader is referred to one of the excellent review articles found in the literature. ${ }^{[33-35]} \mathrm{A}$ large number of papers exist on $\mathrm{ZnO}$ nanoparticles, in particular on nanorods. The majority of examples and nanoparticles exhibit cross-sections (perpendicular to $c$ ), that exhibit the same symmetry as the underlying crystal system, six-fold rotational symmetry in the case of Wurtzite. ${ }^{[36-41]}$ Scheme 1 demonstrates, why it is so difficult to deviate from the hexagonal shape/cross-section.

The surface corresponding to the (100) lattice plane (respectively [10 $\overline{1} 0]$ ) is symmetry-equivalent to the (010) (respectively [0110]) surface. Thus, the interaction with ligands and growth rates should always by identical. The key to the triangular morphology is to break the symmetry of the six equivalent facets of $\mathrm{ZnO}{ }^{[9]}$ There are only few reports in literature, which show this is possible for Wurtzite-based materials deposited on solid substrates. ${ }^{[42-54]}$ In their study on MBE (molecular beam epitaxy) of $\mathrm{GaN}$ on GaN substrates Xie et al. showed that a hexagonal symmetric bilayer of the cation and the anion exhibits two types of alternating step edges: ${ }^{[42]}$ Edge-Type A with two dangling bonds and Type B with only one dangling bond (Scheme 1b). Thus, the two edge types are kinetically distinguishable. Type A step edges are less stable and exhibit a higher growth-rate, which leads to an intrinsically anisotropic growth at that "bilayer-state" of the crystal. Consequently, Type A edges grow faster than Type B edges, which inevitably results in the formation of a triangular shaped bilayer (Scheme 1c). Once this triangular bilayer has formed, all side edges are again kinetically equivalent and a return to a hexagonal shape is less likely to happen. Related results, so called "nanocastles" have been achieved via vapor deposition of $\mathrm{ZnO}$ on GaN substrates by Wang et al. ${ }^{[46]}$ In 2010, Chen et al.

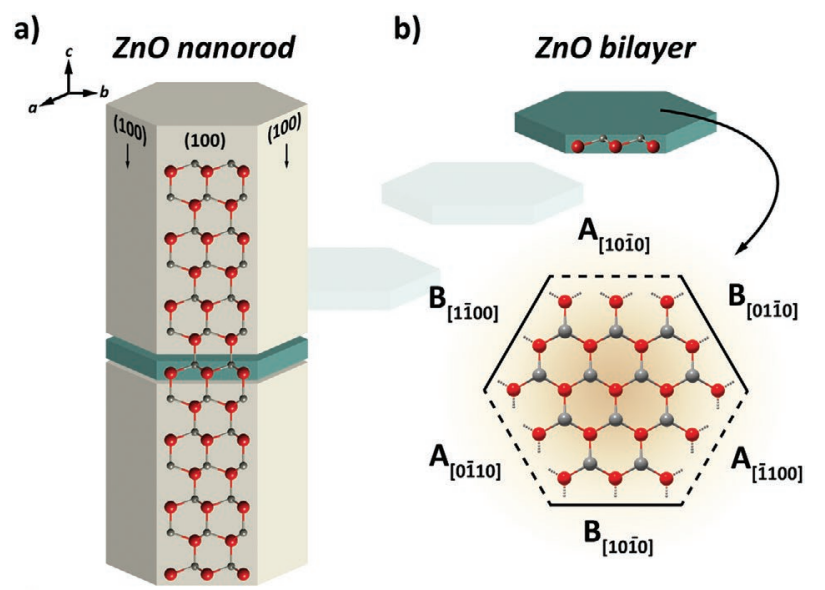

c)

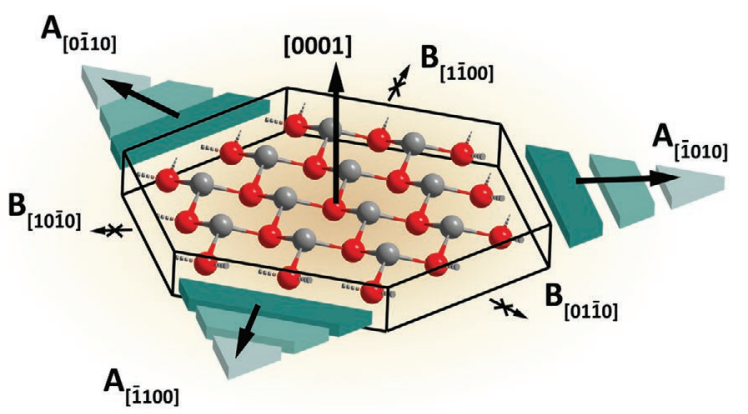

Scheme 1. a) Representation of a hexagonal prismatic $\mathrm{ZnO}$ nanorod with the crystal structure shown on the front facet. b) Bilayer slice of $\mathrm{ZnO}$ exhibiting alternating A-type edges with two dangling bonds per atom and B-type edges with only one dangling bond per atom in growth direction. C) Demonstration of the bilayer symmetry break and the resulting formation of a triangular symmetry.

showed that the return from an initially grown triangular shaped nanoparticle to a hexagonal morphology is indeed possible, and is dependent on the growth kinetics in c-direction..$^{[51]}$

The drawback in the mentioned, substrate-based approaches is of course, the particles are attached to the base-layer. This way it is not possible to obtain colloidal dispersions. However, colloidal particles are needed, if one wants to study the influence of the mentioned symmetry-break on SN self-assembly and on property deviations in the resulting superstructures and particle-based materials. The latter remarks define the goals for the current contribution.

It is important to mention that a change in shape does not necessarily induce a change in symmetry of the corresponding self-assembled superstructure. This contradiction is revealed when looking at the rotational symmetry of spherically- $\left(\mathrm{c}_{\infty}\right)$ and hexagonally $\left(\mathrm{c}_{6}\right)$ shaped nanoparticles. They can obviously be distinguished from each other. However, 2D-superstructures build from these different shapes feature related symmetry attributes. It is known that spherically and hexagonally shaped particles, form identical hexagonally closed packings with $A B A B$ structure. ${ }^{[41]}$ Because a hexagon can be deconstructed into six triangles, one might expect that there will also be no difference in superstructure symmetry. Since we gained first indications that a trigonal shape is possible for $\mathrm{ZnO}-\mathrm{SNs}$ back in 2011, ${ }^{[55]}$ we want to answer the latter question in more detail here. 

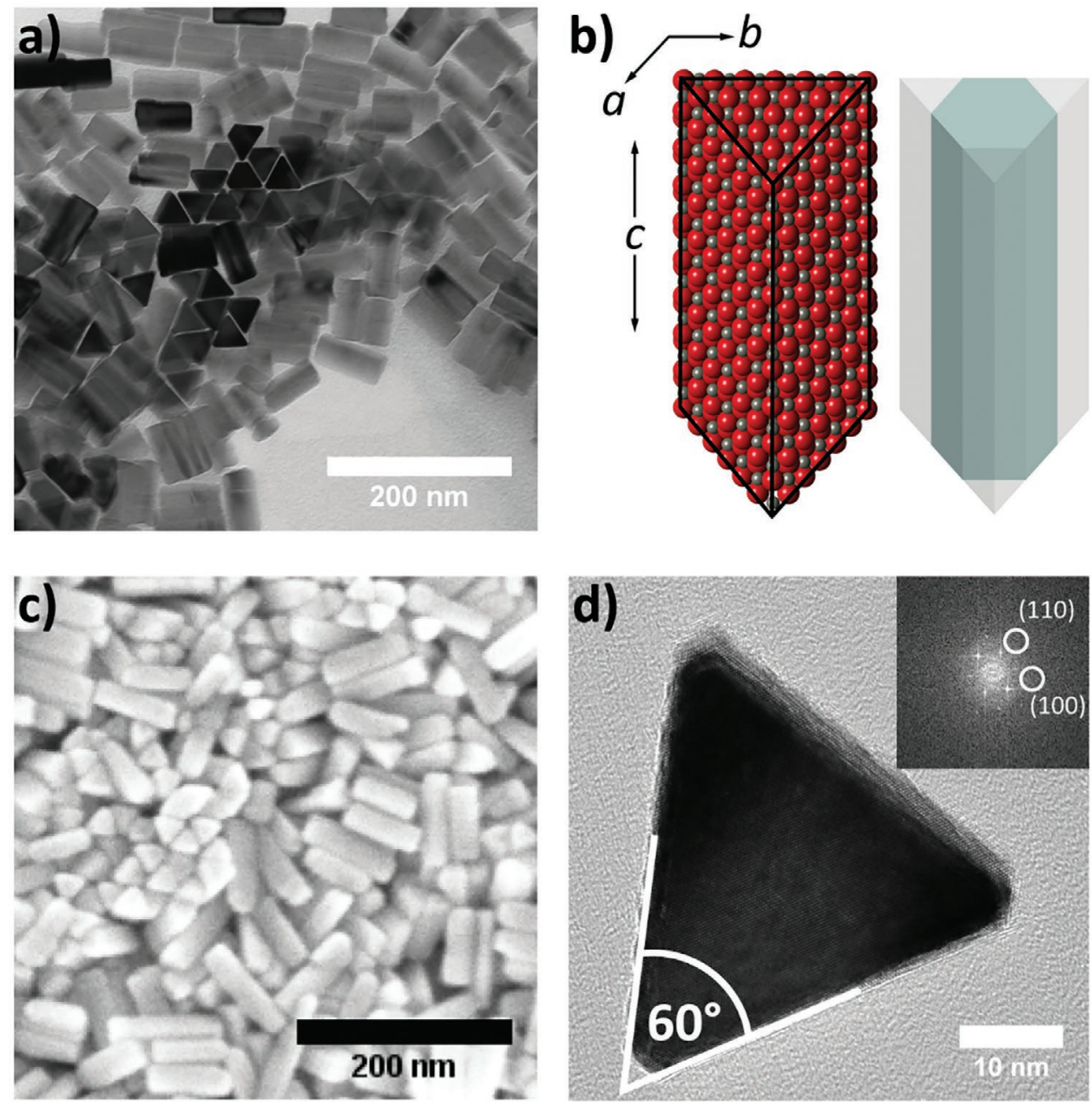

Figure 1. a) TEM image of $\mathrm{ZnO}$ nanorods with triangular cross section. b) Schematic depiction of the morphology and the underlying hexagonal symmetry. c) SEM image of ZnO nanorods with triangular cross section. d) HR TEM image of a single nanoparticle with perfect $60^{\circ}$ and FFT inset.

\section{Results and Discussion}

\subsection{Cooperative Ligand-Induced Symmetry Break}

It was hypothesized in the past, ${ }^{[55]}$ but never convincingly demonstrated that a deviation from the hexagonal shape is possible during a colloidal synthesis, despite there is no solid substrate as a factor for the formation of trigonal shapes. Transmission (TEM) and scanning (SEM) electron microscopy data of nanoparticles prepared as described in detail in the Supporting Information are shown in Figure 1. The TEM image in Figure 1a reveals the uniform, trigonal prismatic morphology of the nanorods. We use the term "trigonal nanorods" for description of those particles in the paper. An alternative denomination "triangular prims" was not used here. The triangle shaped cross-section is clearly evident from the nanorods, that are oriented with the $c$-axis perpendicular to the TEM-grid. A schematic depiction of the triangular nanoprisms is given in Figure 1b. The SEM image in Figure 1c further confirms the triangular prismatic morphology of the $\mathrm{ZnO}$ nanorods. The particles exhibit straight edges with nearly perfectly flat ends. The high-resolution (HR) TEM image in Figure 1d presents the perfect $60^{\circ}$. The six hexagonally arranged spots of the fast Fourier transformation (FFT) inset in Figure 1d fit well to the powder X-ray diffraction (PXRD) measurement of the nanoparticles in Figure S1a (Supporting Information). All reflexes can be assigned to Wurtzite $\mathrm{ZnO}$ (space group P63mc). In accordance with the low size distribution measured by dynamic light scattering (DLS) (Figure S1b, Supporting Information), manual measuring of TEM images yielded a very low polydispersity, with PDIs of less than $10 \%$ for both the length and the width of the triangular nanorods (Figure S1c, Supporting Information).

The capping agents used in the described method, are related to a commercially available emulsifier ("P3P" = polyglyceryl-3-polyricinoleate), which is known in the cosmetic industry and also for the preparation of other $\mathrm{ZnO}$ nanoparticles. ${ }^{[5-57]}$ From a chemical analysis of P3P, we learned that it actually is an oligomeric compound with glycerol-oleate-esters of various composition (see Figure S2 in the Supporting Information). It is obviously not clear, which of the constituents of P3P is responsible for the emergence of the trigonal shape of the $\mathrm{ZnO}$ nanorods, neither is the formation mechanism clear at all. Nevertheless, the main affinity towards $\mathrm{ZnO}$ is supposed to lie in the structure-motive of the glyceryl-moiety and hence, a dependency of the number of free $\mathrm{OH}$-groups can be assumed. Therefore, to investigate the influence of the glycerol moiety 
on the shape of $\mathrm{ZnO}$, we use three different ligands, namely glycerol-monooleate (mono-Ol), 1,3-glycerol-dioleate (di-Ol), and glycerol-trioleate (tri-Ol) in the synthesis of $\mathrm{ZnO}$ nanoparticles. The ligands differ by the grade of esterification of the glycerol moiety. Mono-Ol features two free $\mathrm{OH}$-groups, di-Ol one and tri-Ol none (see Figure S2, Supporting Information). Because neither one of the components mono-Ol, di-Ol, tri-Ol are commercially available in pure form, they were synthesized by us (see experimental section). We can now investigate the influence of the isolated ligand compounds and also of their mixtures on the $\mathrm{ZnO}$ morphogenesis.

We prepared three different batches of $\mathrm{ZnO}$ nanoparticles, with $100 \%$ mono-Ol $[\mathrm{M}], 100 \%$ di-Ol [D] and $100 \%$ tri-Ol $[\mathrm{T}]$ respectively. A reliable tool to investigate the general affinity of ligands towards the $\mathrm{ZnO}$ nanoparticles is given by infra-red spectroscopy (IR). Interactions of ligands with the nanoparticle surface induce a shift of the signal of a certain stretching bond (e.g., $\mathrm{C}=\mathrm{O}$ ) toward different wavenumbers. To probe the quality of the interaction with the $\mathrm{ZnO}$ surface we compare the original (noncoordinated) with the shifted (coordinated) signal. Figure S3d (Supporting Information) presents the ratio $(r)$ of the normalized relative intensity (norm. $I_{\text {rel. }}$ ) of the coordinated $\mathrm{C}=\mathrm{O}$ stretching bond $\left(\approx 1560 \mathrm{~cm}^{-1}\right)$ with the norm. $I_{\text {rel. }}$ of the noncoordinated $\mathrm{C}=\mathrm{O}$-stretching bond $\left(\approx 1740 \mathrm{~cm}^{-1}\right)$. We conducted identical washing cycles for each nanoparticle batch and measured an IR spectrum after each washing step. After the first washing step the ratio is below one for all three batches $[\mathrm{M}](r=0.9)$, [D] $(r=0.8)$ and [T] $(r=0.7)$, indicating a higher percentage of noncoordinated ligand. After the second and third washing step, excess ligand is washed of and the ratio is significantly higher than one for $[\mathrm{M}](r=1.5)$ and $[\mathrm{D}](r=1.3)$, signifying a higher percentage of coordinated ligand. For [T], $r$ remains at 1.1, indicating nearly same percentages for coordinated and non-coordinated ligand. These results suggest that the interaction with the $\mathrm{ZnO}$ surface is strongest for mono-Ol, medium for di-Ol and relatively unspecific for tri-Ol. Moreover, these results imply that the number of free $\mathrm{OH}$-groups of the ligand can be correlated to the affinity toward the $\mathrm{ZnO}$ surface. Due to their different affinity toward the $\mathrm{ZnO}$ surface, the ligands should exhibit different growth-directing properties.

However, there is still an explanation missing, how can there be a different interaction of one of those ligands with faces A (Scheme 1) compared to the B-faces? From literature we know that the formation of the triangular shape takes place at an initial phase of growth. ${ }^{[42,47,51]}$ Consequently, a pivotal step has to be efficient stabilization during this critical phase. The in situ analysis of such initial phases with conventional analytical methods like TEM faces serious problems like sample decay/conversion during preparation. Hence, they lack in accuracy and are unsuitable to investigate the early stages of growth. An appropriate alternative to approach such early phases can be done by computational methods. Therefore, we calculated the initial hexagonal-shaped $\mathrm{ZnO}$ bilayers with the ADF (Amsterdam density functional) code implemented in the SCM (Software for Chemistry \& Materials B.V.) suite on PBE (Perdew-Burke-Ernzerhof) and nonrelativistic double zeta level of theory. ${ }^{[58,59]}$ First, geometry optimizations were performed to reorganize the surfaces. Vibrational analyses were performed on the structures to check for true minima. Subsequently, the lowest unoccupied molecular orbitals (LUMOs) are visualized by the SCM suite. We performed computational simulations of the initial five bilayers of $\mathrm{ZnO}$ (001). The results presented in Figure 2 show a clear dependence of the electronic structure at the (100)-edges on the number of $\mathrm{ZnO}$ (001) layers.

Presented in Figure 2, a freestanding single bilayer is actually heavily distorted without the supportive rigidity of a substrate,
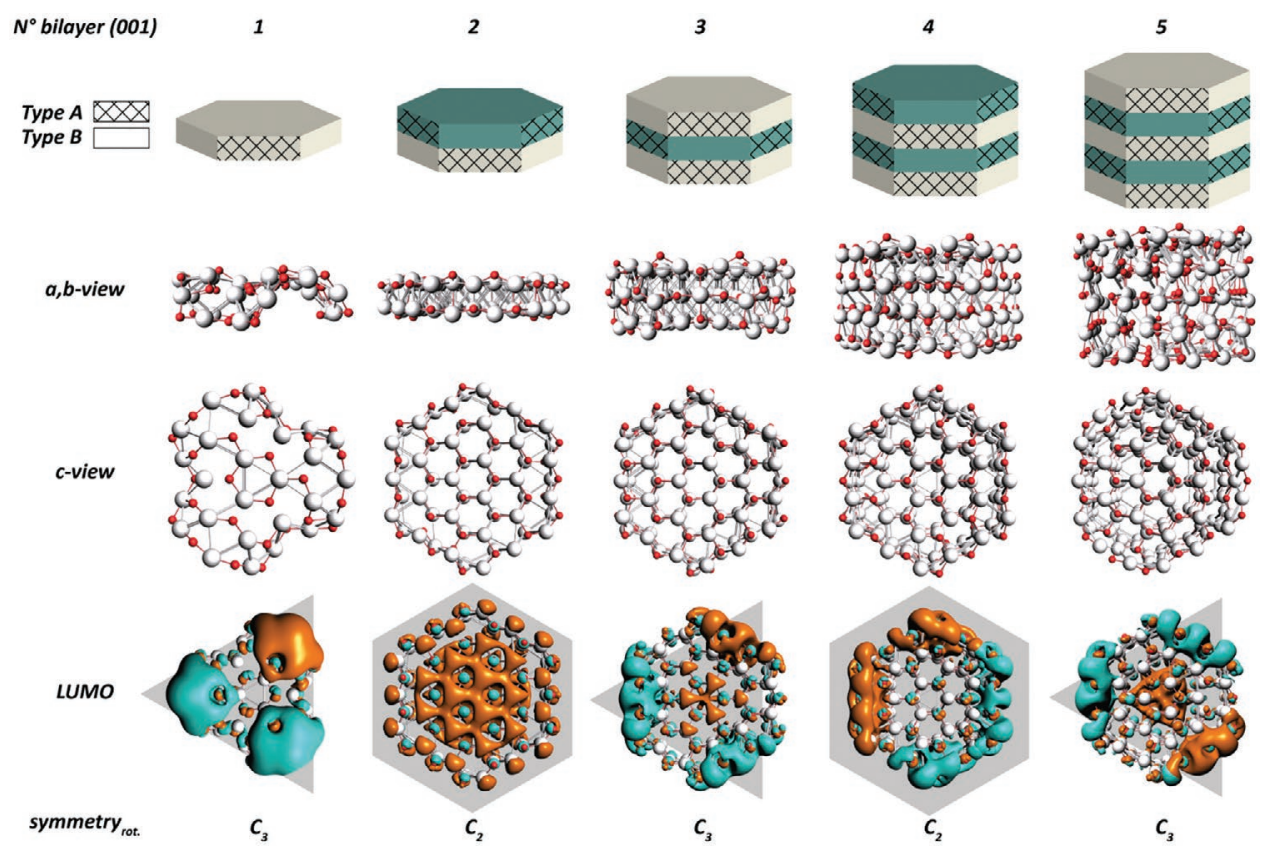

Figure 2. Schematic depictions of the initial five bilayers of $\mathrm{ZnO}$; Type-A edges are represented by criss-crossed facets. Computational simulations of the initial five bilayers of $\mathrm{ZnO}$, seen from $a, b$ - and $c$-direction. Distribution of the electronic structure, with respect to the number of bilayers and the resulting symmetry of the LUMO. 
neglecting a clear differentiation of the two different edge types A and B. Nevertheless, when looking at the calculation of the distribution of the electronic structure, there is already a distinct anisotropy observable. The LUMO is distributed in a triangular fashion resulting in $\mathrm{C}_{3}$ symmetry of the structure including the electronic structure. Two bilayers of $\mathrm{ZnO}$ are able to form a planar sheet, with distinct facets. Since every additional bilayer is rotated $60^{\circ}$ with respect to the previous layer, the Type A edge of the second layer is not directly above the first one, resulting in "Type A-B" and "Type B-A" facets. Therefore, in the case of two bilayers, every (100) facet exhibits the same electronic structure. Hence, the LUMO is evenly spread across every facet leaving no anisotropy in the (100) facets. The addition of a third bilayer of $\mathrm{ZnO}$, which is again rotated $60^{\circ}$ with respect to the previous layer, changes the situation dramatically. The different facets can now be deduced as "Type A-B-A" and "Type B-A-B". Now, an anisotropy in the electronic structure of the alternating (100) facets can be observed. The electronic structure is distinctively concentrated on three of the six facets of the structure indicating a triangular $\mathrm{C}_{3}$ symmetry. When adding a fourth and fifth bilayer to the structure, similar results as before can be observed. With four bilayers, we observe a relatively even spread LUMO on all of the six facets, while the electronic structure is again locally concentrated on only three facets for five bilayers of $\mathrm{ZnO}$.

The obtained results for the modeled structures illustrate that for an even number of $\mathrm{ZnO}$ bilayers, the six (100) facets are electronically symmetric and no triangular growth is possible. For every odd multiple of bilayers, the electron structure is distinctively positioned on alternating edges indicating a triangular $\mathrm{C}_{3}$ rotational symmetry and the possibility of triangular growth. Hence, we can state that the formation of a triangular shape is not restricted to only one bilayer of $\mathrm{ZnO}$. We assume that small clusters with an odd number of bilayers are involved in the nucleation and growth mechanism of the triangularshaped $\mathrm{ZnO}$ nanorods. The difference in the electronic structure could lead to a preferred growth of one facet over the other. Therefore, the initially hexagonal-shaped nucleation seeds turn into triangular ones.

It can also be assumed that with every additional bilayer the difference in alternating facets continuously decreases. The growth rate in the (001) direction must therefore be low so that a triangular seed is formed first. Otherwise, hexagonal-shaped nanorods would also be visible.

It can also be concluded, a straight forward approach to investigate the transition from six-fold to three-fold symmetry, is to examine the occurrence of $120^{\circ}$ - and $60^{\circ}$-angles (see also Scheme 1). The abundance of those angles directly refers to either a hexagonal or a triangular morphology. To assign a value for the transitory morphology from a hexagonal to a triangular symmetry we introduce $Q_{\text {morph. }}$ as the quotient of the quantity of $60^{\circ}$-angles divided by the quantity of $120^{\circ}$ :

$Q_{\text {morph. }}=\frac{N_{60^{\circ}}}{\frac{1}{2} N_{120^{\circ}}}$

The value for $\mathrm{N} 120^{\circ}$ has to be divided by two, since the maximum number of $120^{\circ}$ per particle (perfect hexagon: six $120^{\circ}$ ) is twice the maximum number of $60^{\circ}$ per particle (perfect triangle: three $60^{\circ}$ ). Figure 3 shows, how the ligands mono-Ol, di-Ol, and tri-Ol and their mixtures affect the morphology of the $\mathrm{ZnO}$ nanoparticles.

Figure 3a shows a Gibb's phase triangle with the three ligands mono-Ol, di-Ol, and tri-Ol at its edges. The position of the dots corresponds to syntheses with different compositions of the used ligands. $Q_{\text {morph }}$. is normalized and represented by the color-gradient bar. The color of the dots in the Gibb's phase triangle represents their position on the color-gradient bar, and hence the morphology of a synthesis with that specific composition of ligands (detailed information on the syntheses composition and the exact values for $Q_{\text {moprh }}$ are given in Table S1 and Figure S4, Supporting Information). A direct look at the different resulting morphologies is given by a selection of colorframed TEM images in Figure 3b1-4. Results of the syntheses with pure ligands $([\mathrm{M}],[\mathrm{D}]$, and $[\mathrm{T}])$ are shown in Figure 3b, images 1-3. As expected from the IR experiments, the differences in size, shape, and dispersity is obvious. With $100 \mathrm{~mol} \%$ mono-Ol (Figure 3b-1), colloidal $\mathrm{ZnO}$ nanoparticles can be obtained, which underlines a distinct affinity of mono-Ol to the $\mathrm{ZnO}$ surface. The particles are single crystalline (Figure S4b2, Supporting Information) and exhibit irregular anisotropic shapes like short rods, platelet-like or intermediate structures. a)

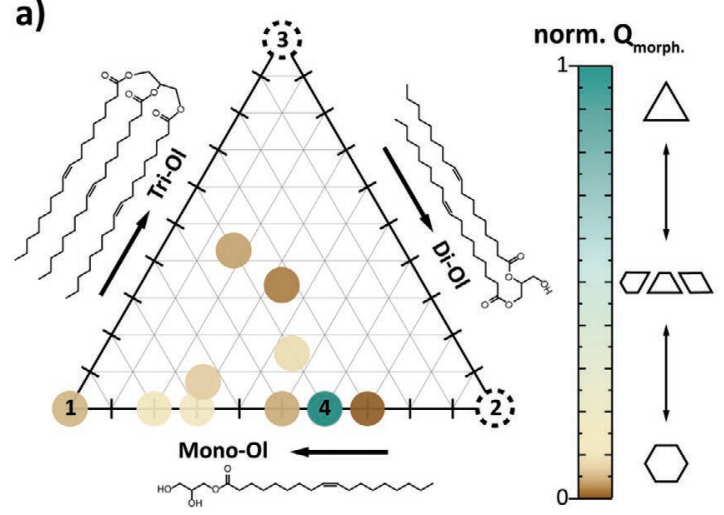

b)

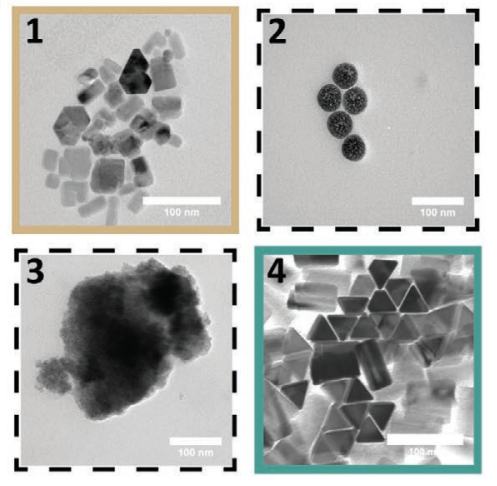

Figure 3. a) Gibbs phase triangle of $\mathrm{ZnO}$ nanoparticles synthesized with different compositions of mono-, di- and tri-Ol, together with a color-gradient bar, representing the value of $Q_{\text {moprh }}$ of the resulting $\mathrm{ZnO}$ nanoparticles. b) TEM images of the synthesized $\mathrm{ZnO}$ nanoparticles, presented with the corresponding color code, scalebars $100 \mathrm{~nm}$. 
Most of the particles have sharp edges and some show first hints of a triangular morphology. Hence, mono-Ol does not only interact with the $\mathrm{ZnO}$ nanoparticles, it also has a distinct influence on the resulting shape and acts as a shape-directing agent. Contrary to this, $100 \mathrm{~mol} \%$ di-Ol leads to a totally different outcome. The resulting $\mathrm{ZnO}$ nanoparticles (Figure 3b-2) are less stable and start to agglomerate rather quickly. Instead of single-crystalline, irregular shaped nanoparticles, di-Ol leads to very uniform, polycrystalline $\mathrm{ZnO}$ nanospheres, with a low size distribution of only $7.1 \%$. Hence, the use of $Q_{\text {morph }}$. is not applicable, as there are neither $120^{\circ}$ - nor $60^{\circ}$ found. Consequently, the dot in the Gibb's phase triangle is not colored, but marked with a dotted frame. The same applies for the corresponding TEM image in Figure 3b-2. Since the nanospheres are present as individual units, it is reasonable that they stem from uniform, monodisperse emulsion droplets. This further implies that the conversion of the single source precursor $[\mathrm{MeZnOR}]_{4}$ into $\mathrm{ZnO}$ takes place within those monodisperse droplets. In accordance with the IR results, di-Ol acts more as a polydispersity-controlling-, rather than a shape direction agent. We can conclude that di-Ol has a significant influence on the texture of the emulsion during the synthesis and is able to portion $\mathrm{ZnO}$ precursor species into equal units. As expected from the IR experiments, tri-Ol (100 mol\%) has the least influence on the resulting $\mathrm{ZnO}$ nanoparticles, which can be seen from Figure $3 \mathrm{~b}-3$. The obtained structures resemble $\mathrm{ZnO}$ particles obtained from syntheses without any added ligand (Figure S4c, Supporting Information). Apparently no individual nanoparticles can be found. Furthermore, the obtained structures consist of agglomerated nanoparticles or grown-together crystallites. Again, the use of $Q_{\text {morph }}$ is not applicable and the corresponding composition is marked with a dotted frame in the Gibb's phase triangle as well as for the TEM image in Figure 3b-3. Tri-Ol neither acts as crystal-growth direction agent, nor does it influence the texture of the emulsion. The negligible influence of tri-Ol on the morphology is not surprising as it exhibits no free OH-group as main interaction moiety.

To achieve the desired $\mathrm{ZnO}$ nanorods with a defined triangular morphology and a low size distribution, it is obvious to utilize a combination of the above illustrated effects of the different ligands. An equimolar combination of all three ligands lead to nanoparticles with sharp edges and an overall more uniform shape (Figure S4b-4, Supporting Information). The $\mathrm{ZnO}$ nanoparticles seem to have improved compared to the syntheses with only one ligand species (Figure 3b1-3). Despite the more defined features, they exhibit nearly solely $120^{\circ}$ at their edges resulting in the common hexagonal shape and a low rating according to $Q_{\text {morph }}$. As shown earlier, monoand di-Ol show stronger interactions towards $\mathrm{ZnO}$ than tri-Ol, which therefore can be neglected. To exploit the shape-directing properties of mono-Ol with the polydispersity-controlling properties of di-Ol a combination of 50:50 mol\% was used (Figure S4b-5, Supporting Information). The obtained nanoparticles are single-crystalline and exhibit an irregular shape with sharp edges. The particles strongly resemble the results obtained from the synthesis with pure mono-Ol (Figure 3b-1), but there are no similarities to the synthesis with pure di-Ol (Figure 3b-2). This implies that the effect of mono-Ol on the $\mathrm{ZnO}$ morphology is too strong compared to effect of di-Ol, which again is in great accordance to the IR experiments shown in Figure S3 (Supporting Information). Consequently, to also utilize the polydispersity controlling effect of di-Ol, its percentage was increased. With a ratio of 42:58 (mono-Ol:di-Ol) samples where obtained, which exhibit the most $60^{\circ}$ angles and therefore show the highest value for $Q_{\text {morph }}$. (Figure 3b-4). The nanoparticles almost exclusively consist of uniform nanorods with a distinct triangular cross-section.

A closer look on the growth of the $\mathrm{ZnO}$ nanoparticles over time during the synthesis is given by the TEM images and the corresponding data in Figure 4. At an early stage of the synthesis $(<60 \mathrm{~min})$ spherical colloids are found, which resemble the spherical particles found in the syntheses with pure di-Ol (Figure 3b-2). These colloids exhibit a very low polydispersity $(\approx 8-15 \%)$ and tend to assemble into closed packings. The quality of these initial $\mathrm{ZnO}$ species is crucial for the outcome of the synthesis, as they are the units containing the $\mathrm{ZnO}$ prephase. Subsequently, under the shape directing influence of mono-Ol, the amorphous $\mathrm{ZnO}$ prephase starts to convert into crystalline $\mathrm{ZnO}(\approx 60-100 \mathrm{~min})$. This is accompanied by a decrease in size of the previously formed spherical species and the appearance of first crystallites (Figure 4a,b). The decrease in size can be explained by the higher density of crystalline $\mathrm{ZnO}$ in comparison to the amorphous prephase. During that stage of the synthesis, the particles exhibit the highest degree of polydispersity of $\approx 20 \%$ in length and $\approx 12 \%$ in width. The growth of the $\mathrm{ZnO}$ nanoparticles into their anisotropic rod-like shape takes place after the conversion from an amorphous into a crystalline phase (<100 min). Indicated by the slope of the graph in Figure $4 \mathrm{~b}$ the growth rate in $c$ direction clearly surpasses the one in $a, b$-direction. After around $100 \mathrm{~min}$, the extension in $a, b$-direction stays constant, while the extension in $c$-direction nearly doubles. This can be attributed to the kinetically favored growth in $c$ direction for Wurtzite systems, which can be utilized to manipulate the aspect ratio (AR) of the nanorods. After around $180 \mathrm{~min}$ the final dimensions of the $\mathrm{ZnO}$ nanorods are reached, as both the length and the width of the particles stay constant. From here on only the polydispersity changes over time, indicating Ostwald ripening processes. Figure S5a (Supporting Information) shows that increasing the reaction kinetics by the temperature, the AR rises linear from 1.7 at $45^{\circ} \mathrm{C}$ to 3.1 at $75{ }^{\circ} \mathrm{C}$. Furthermore, the AR can be manipulated by varying the addition rate of the $\mathrm{ZnO}$ precursor. A slow addition rate of $0.05 \mathrm{mmol} \mathrm{min}^{-1}$ leads to an AR of 3.1, while a faster addition of $0.15 \mathrm{mmol} \mathrm{min}^{-1}$ results in an AR of 1.8 (Figure S5b, Supporting Information). Surprisingly, in both cases the width of the $\mathrm{ZnO}$ nanoparticles stays rather constant.

We can now formulate an educated guess about the mechanism of the formation of $\mathrm{ZnO}$ nanorods with trigonal crosssection. The role of di-Ol is the stabilization of small particles, which contain an amorphous $\mathrm{ZnO}$ precursor-phase. Because the extension of the particles is so small, there is only a limited number of ZnO-layers stacking on each other. According to Figure 2, this enhances the chances for the symmetry break. Mono-OL interacts with the surfaces and, thus, locks the symmetry break. The thin, trigonal nanoplates, respectively adjacent particles fuse (Figure 4a; second image), and as a consequence the trigonal particles grow. 


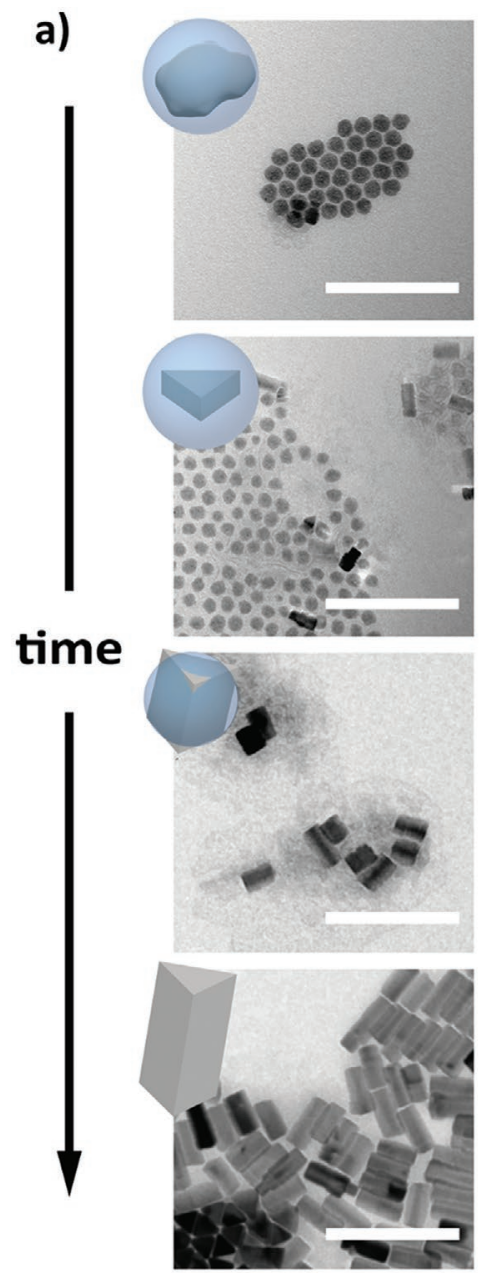

b)

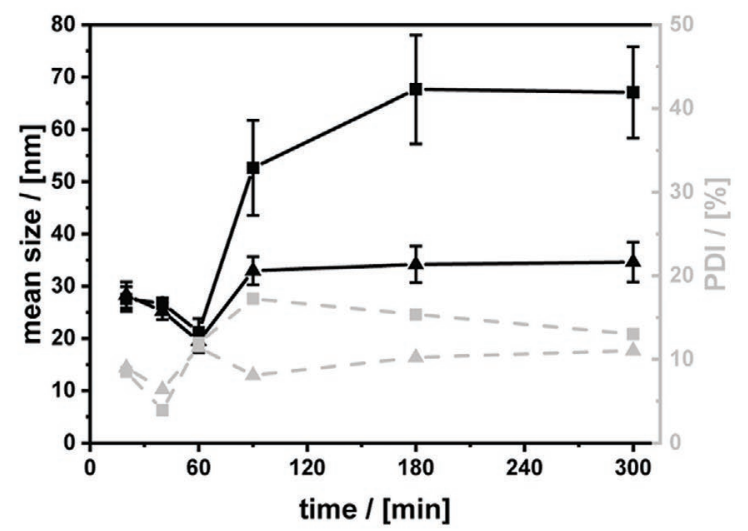

c)

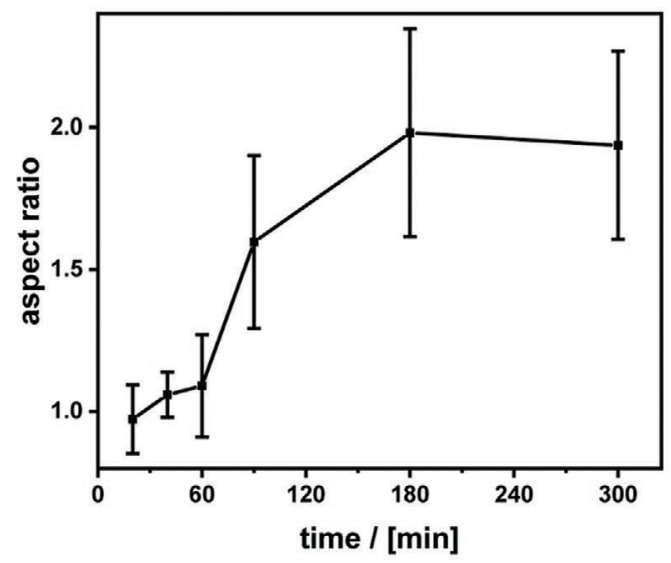

Figure 4. a) TEM images taken at different times during the growth of the trigonal $\mathrm{ZnO}$ nanorods, scalebars represent $200 \mathrm{~nm}$. b) Mean size of the nanorods (length $=$ squares; triangles $=$ width) in black and polydispersity in grey against the time during the synthesis. c) Evolution of the aspect ratio of the nanorods.

\subsection{Triangular Nanorods as Building-Blocks and Shape Symmetry Correlations}

$\mathrm{Up}$ to this point, monodisperse trigonal $\mathrm{ZnO}$ nanorods are available (Figure 1) and, we have understood how the necessary symmetry break occurs on the level of an isolated particle. We can now investigate, if the "transition"/symmetry break from hexagonal to trigonal shapes has an influence on the selfassembly behavior as well and, thus, also on properties of particle-based materials.

First, it is necessary to assemble the trigonal (and hexagonal) $\mathrm{ZnO}$ colloids to extended periodic arrays (Figure 5). This has been done using the method presented by Dong et al., ${ }^{[12]}$ which we already employed in previous works to assemble hexagonal ZnO nanoplates. ${ }^{[41]}$ Herein, a defined amount of NP dispersion is spread onto diethylene-glycol (DEG) in a small glass petridish. After evaporation of the solvent, the residual NPs form a film on the DEG surface, which can be transferred to arbitrary surfaces. The SEM image in Figure 5a shows a large-scale section of the triangular $\mathrm{ZnO}$ nanorods, standing perpendicular on a silicon substrate. As indicated by the schematic inset in the top-right corner, an interlocking architecture is extended in all directions parallel to the substrate, creating a closely packed superlattice. One can observe domains in which the particles form hexagonally ordered packings that span over several hundred nanometers. Although the particles are arranged in a 2D closed packing, one can clearly observe a certain porosity within the structure. Side-views of multilayered superstructures are shown in Figure S6 (Supporting Information). The SEM images show long range, ladder-like arrays, again spanning over several hundred nanometers.

More details about the packing of the SNs can be obtained from TEM measurements. The close up TEM image in Figure 5b shows, that via interlocking at the (100) facets the particles stack up into a staggered repetitive pattern. The black lines in the image show different measured distances $d_{\text {theo }}$. (70.6 nm), which refers to twice the width of a single nanorod and $d_{\text {real }}(63.5 \mathrm{~nm})$ (dotted). As there is a significant difference of $\approx 10 \%$, the particles seem to be in closer proximity to each other, than they should. A more transparent view of the structure is given in Figure 5c. The TEM high angle annular dark field (HAADF) image clearly shows that the particles are single 

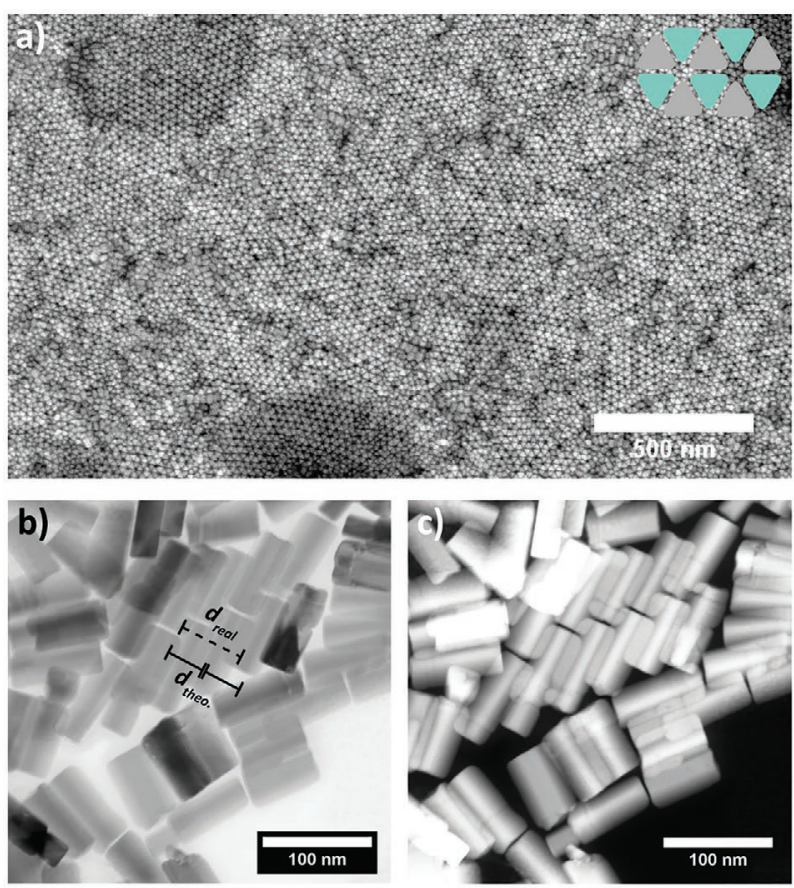

d)

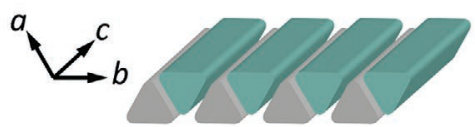

Figure 5. a) SEM image of long range ordered superstructures of standing $\mathrm{ZnO}$ nanorods. b) TEM image of interlocked triangular $\mathrm{ZnO}$ nanorods. c) HAADF TEM image of the same spot. d) Schematic depiction of the interlocking assembly and corresponding crystallographic directions.

units with sharp edges and no sintering effects have occurred. Furthermore, one can see a distinct overlap between every two particles, which fits to the difference in the distances $d_{\text {theo }}$. and $d_{\text {real }}$. Thereby, we can conclude that the difference is caused by an alternating turn of $180^{\circ}$ along the $c$-axis with each next particle during the assembly. A tilted view of this assembly architecture is schematically drawn in Figure 5d.

To conduct a profound comparison of the two different shapes (hexagons and triangles) we synthesized common hexagonal $\mathrm{ZnO}$ nanorods in addition to the triangular $\mathrm{ZnO}$ nanorods after a protocol which we published in previous work. ${ }^{[41]}$ Similar to the triangular $\mathrm{ZnO}$ nanorods, these hexagonal nanorods were also self-assembled via the above presented method by Dong et al. ${ }^{[12]}$ (see Figure S7, Supporting Information).

As mentioned above, the threefold symmetry of the nanoparticles should have a significant impact on the symmetry of the assembled superstructures. Triangular particles are able to build superstructures related to the HCP, but exhibit distinct symmetry differences. These symmetry-differences are described by the so-called Schläfli mosaic-tiling nomenclature which can be applied to any regular polygon tiling. ${ }^{[60]}$ The symmetry one individual polygon is given by the number of its corners $(X)\left(X_{\text {triangle }}=3, X_{\text {hexagon }}=6\right)$. The symmetry of the corresponding polygon-tiling is given by the number of individual polygons, which concur at their corners $\mathrm{X}^{\mathrm{Y}}$, the so-called vertex points. For a triangle, six individual triangles can concur at each corner, resulting in a $3^{6}$ symmetry. For a hexagon, three individual hexagons can concur at each corner, resulting in a $6^{3}$ symmetry. To highlight the difference between periodic arrays formed by hexagonal nanorods and trigonal nanorods, we compare the symmetries of both hexagon- and trianglebased superstructures in Figure 6.

TEM images of superstructures formed by common hexagonal $\mathrm{ZnO}$ nanorods are shown in Figure 6a and superstructures formed by triangular $\mathrm{ZnO}$ nanorods in Figure 6b. In both cases the nanorods stand perpendicular to the substrate (TEM grid), presenting their (001) facet. Indicated by the black hexagon in the schematic depictions of the two superstructures, both exhibit a hexagonal symmetry and belong to the P6m symmetry group. Nevertheless, the difference between these two mosaic tilings is clearly visible as shown by the gray-colored particles in both structures (Figure 6a,b). As mentioned above three nearest neighboring hexagons meet at the corner of each other, giving the hexagonal tiling a $6^{3}$ symmetry, whereas in Figure 6b, six nearest neighboring triangles concur at the tip of each other, resulting in a $3^{6}$ symmetry. This altered symmetry is clearly referable to the difference in morphology and has an impact on the point lattice symmetry of the superstructures (Figure 6c). The point lattice symmetry on the left side (ochre color) resembles the simple 2D closed packing and fits to superstructures formed by spherical particles, as well as in our case hexagonal nanorods. The right side (cyan color) resembles the symmetry of the lattice formed by the triangular shaped nanorods. The structure obviously differs from the closed packing, as there are empty spots, which are indicated by the gray circles on the right side of Figure $6 c$. These empty spots are symmetrically ordered and span a periodically ABAB-vacancy superlattice. Within the nanoparticle superstructure, those vacancies are located where the tips of six triangles concur. Since the superstructure is made of vertically aligned nanorods, those vacancies exhibit an elongated channel-like shape. The formation of meso-scale voids with a diameter of $\approx 6 \mathrm{~nm}$, can be seen as an emergent phenomenon, which is possible only for the trigonal nanorods but not the hexagonal particles (Figure 6d).

\subsection{Cooperative Optical Properties}

Nanoparticle based materials in from of ordered assemblies may have potential for applications in optics, because the size of the constituents and the periodicity of the superlattice are similar to an optical wavelength. Therefore, nontrivial near-field effects beyond the refractive index of the constituents can be expected. In order to investigate the optical properties of our structures, we invoke numerical simulations with the finitedifference time-domain method (FDTD, Lumerical Inc.). The model structures under investigation are regular assemblies of hexagonal and trigonal nanorods; see Figure 7. The side lengths of the hexagonal and trigonal nanoparticles are 16 and $40 \mathrm{~nm}$, respectively. These values lead to a similar effective surface area in both cases in order to produce an instructive comparison. The height of both structures is $80 \mathrm{~nm}$. The electric and magnetic field vectors are calculated for an incoming plane wave of a wavelength of $575 \mathrm{~nm}$ that impinges along the $z$ axis. The numerical mesh size is $0.5 \mathrm{~nm}$ and the boundary conditions 
a) hexagonal tiling $\rightarrow 6^{3}$

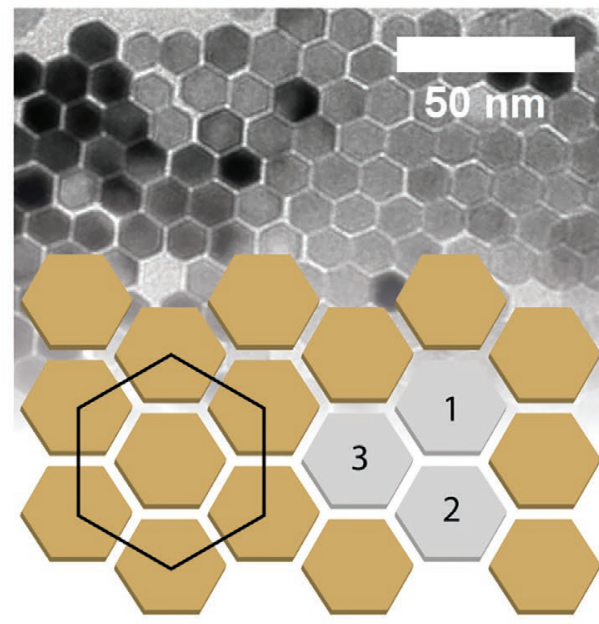

c) point lattice symmetry

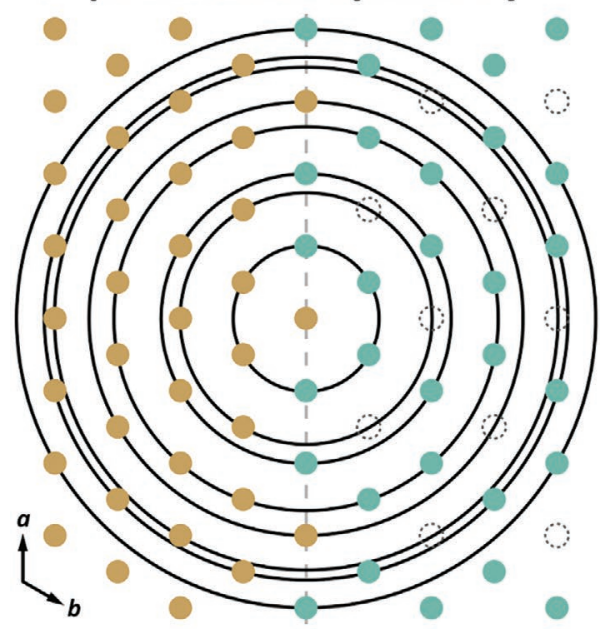

b) triangular tiling $\rightarrow 3^{6}$

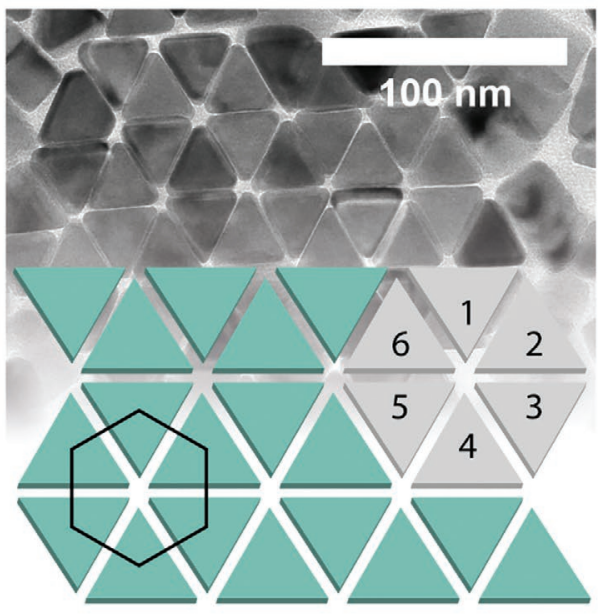

d)

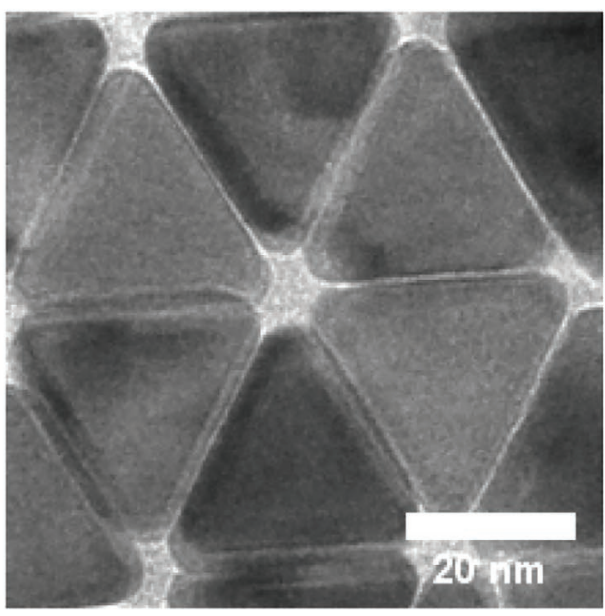

Figure 6. a) Periodic superstructure of common hexagonal ZnO nanorods and corresponding schematic depiction of the superstructure. b) periodic superstructure of triangular $\mathrm{ZnO}$ nanorods and corresponding schematic depiction of the superstructure. c) Comparison of the respective point lattice symmetry of both superstructures. d) Excerpt showing the formation of voids in the superstructure of trigonal nanoparticles.

along the $x$ and $y$ axes are set to periodic, in order to simulate an infinite area of self-assembled nanostructures. The refractive index of $\mathrm{ZnO}$ is taken from Querry. ${ }^{[61]}$ The magnitude of the electric field, averaged over time, is evaluated at $z=40 \mathrm{~nm}$, a position in the middle of the nanostructures, where the maximum field enhancement in the gaps can be expected. Figure 7 shows in the upper two panels the structures and in the middle and lower panels the resulting near-field enhancements. Blue colors indicate attenuations and red colors reveal enhancements. Although both superlattices are composed of a similar amount of $\mathrm{ZnO}$ material, their manipulation of the electromagnetic fields differs substantially due to the altered symmetry of the constituents and lattice. For a $0^{\circ}$ polarization and incident electric field vectors along the $x$-axis (middle panel), the field enhancements in the hexagonal nanorods (left middle panel, Figure 7a) are interrupted and periodically displaced. In contrast, the distribution of the field enhancement in the trigonal nanorods (right middle panel, Figure $7 \mathrm{~b}$ ) is orthogonal to the incident polarization and consists of almost uninterrupted lines in form of a wire grid. When changing the polarization by $90^{\circ}$ (see lower panels), the patterns of the field enhancements change substantially and we now see a regular wire grid for the hexagonal shapes (lower left panel) and a distorted 2D grid for the triangular structures (lower right panel). In all cases, the peak field enhancement is $\approx 1.8$.

We conclude that our regular nanorod assemblies confine light at subwavelength dimensions with distinct and regular near-field patterns over a macroscopic area that is only limited by the physical size of the assembly. This result is reminiscent of metamaterials, that is, nanostructured antenna arrays that modify an incoming optical radiation in ways that are impossible to achieve with natural materials. ${ }^{[62-64]}$

It can be concluded that special optical materials may emerge from the scalable and regular array of sub-wavelength structures 

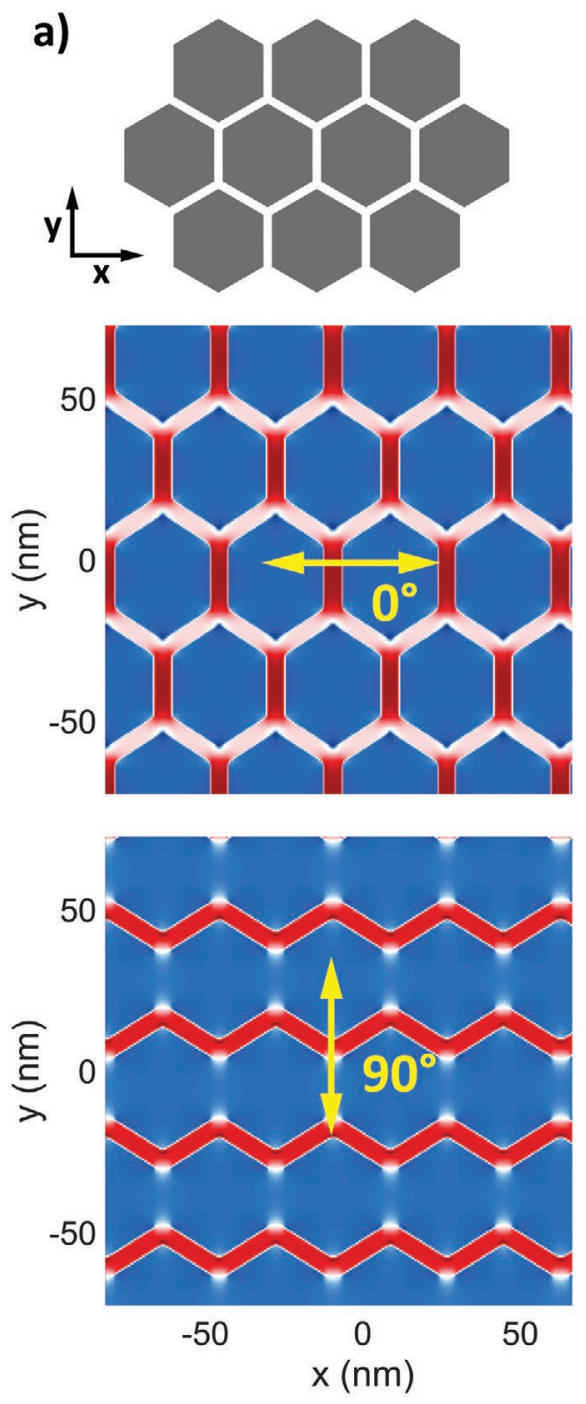
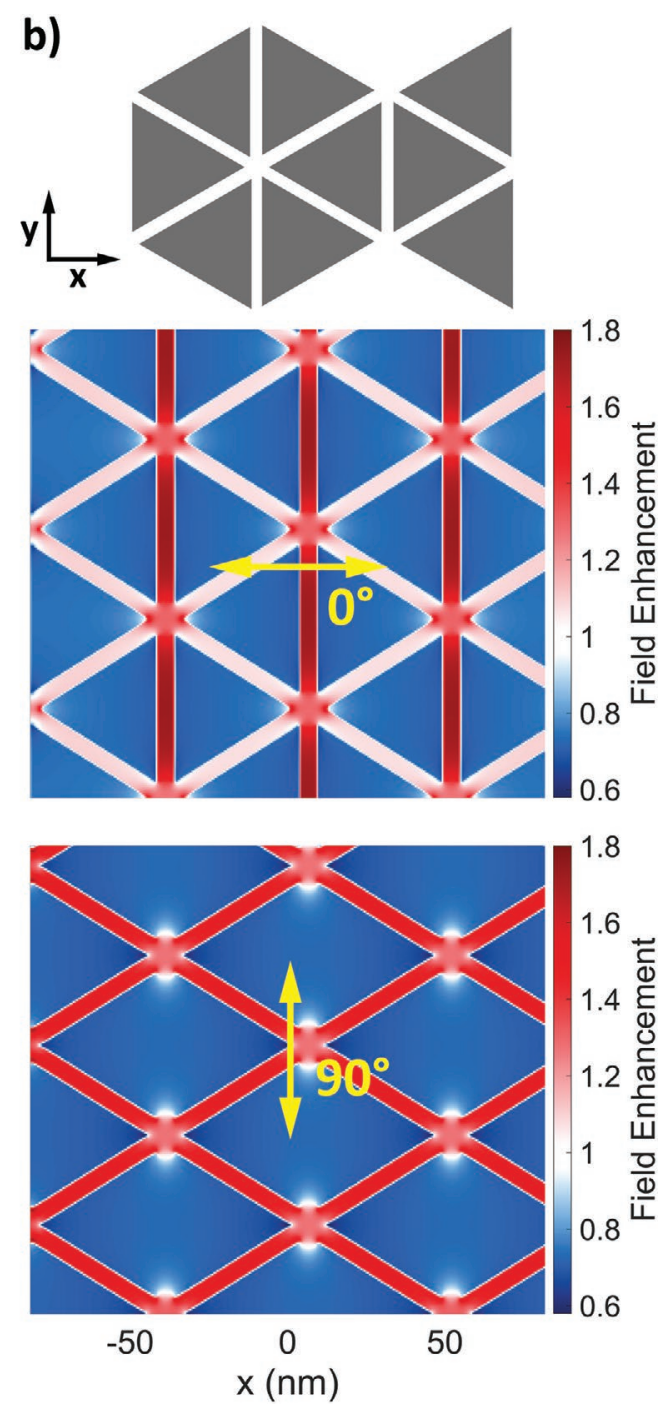

Figure 7. Numerical simulations of optical near-fields in periodic ZnO nanoparticle superlattices. a) Polarization-angle dependent field enhancements of hexagonal $\mathrm{ZnO}$ nanorod structures. b) Polarization-angle dependent field enhancements of trigonal ZnO nanorod structures.

with functional near-field response. The reported polarizationtunable and regularly distributed optical field enhancements might also serve for plasmon-enhanced Raman spectroscopy, ${ }^{[65]}$ facilitate the optical sensing of low amount of substances ${ }^{[65,66]}$ or localize photocatalytic activity to desired regions in space with nanometer precision. Experimental investigations of spatiotemporal nearfields with attosecond electron microscopy ${ }^{[67]}$ or Aharonov-Bohm diffraction ${ }^{[68]}$ might provide further insight, in particular on the role of round edges and imperfections, but the reported proofof-concept simulations already show that regular or semiregular nanoparticle arrays under symmetry control with ligand chemistry might be a useful tool for creating nanophotonic functionality with a scalable synthesis approach.

\section{Conclusion}

Nanoparticle-based materials feature the properties of individual nanoparticle constituents, as well as cooperative effects, if the building-blocks are arranged in close, ordered proximity. Hence, the morphology of the nanoparticles and the symmetry of the corresponding superlattice play a major role in defining and the properties of such materials. In the current work, the impact of a symmetry break of individual nanoparticles on the symmetry and properties of respective self-assembled superstructures is investigated. Two different anisotropic morphologies of $\mathrm{ZnO}$, hexagonal and triangular nanorods, are used to highlight that not every shape is able to induce such a consecutive change in the symmetry of self-assembled superstructures. Therefore, a microemulsion-based route toward colloidal $\mathrm{ZnO}$ nanorods with triangular cross-section was established and the formation mechanism of this uncommon shape was analyzed via computational and experimental methods. In contrast to examples from literature, we found that the necessary symmetry break to obtain triangular $\mathrm{ZnO}$ morphologies is not restricted to just one "bilayer-state," but is possible for the first few bilayer-multiples of $\mathrm{ZnO}$ with odd numbers. In that case, the (100) facets exhibit an alternating electronic structure, 
enabling the triangular growth of otherwise equivalent hexagonal symmetric facets. Instead of a solid substrate, this initial $\mathrm{ZnO}$ state was efficiently stabilized by a defined combination of glycerol-monooleate (mono-Ol) and 1,3-glycerol-dioleate (di-Ol) as ligands in a collaborative ligand interaction. Herein, monoOl was identified as being the active growth directing agent, while di-Ol affects the texture of the micro-emulsion, enabling monodisperse droplets of amorphous $\mathrm{ZnO}$ prephase. This collaborative ligand approach resulted in the growth of exceptionally uniform $\mathrm{ZnO}$ nanorods with distinct triangular crosssection and very low polydispersity $(<10 \%)$.

Consequently, the impact of the triangular shaped $\mathrm{ZnO}$ nanorods on the formation, symmetry and properties of 2D nanoparticle superstructures was investigated and compared to superstructures built from common hexagonal $\mathrm{ZnO}$ nanorods. For the common hexagonal symmetric nanoparticles, we observed a hexagonal closed packing, similar to simple spherical particles. In the case of triangular nanorods, the shape induces a symmetry break in the superstructure accompanied by the appearance of new emergent properties. One effect was the formation of mesoscale voids. In addition, numerical simulations of optical near fields revealed that the altered symmetry of the nanoparticle superlattice has a significant effect on the interaction and propagation of electric fields through the structures, which implies high potential for the application as optical nanoparticle-based materials. Furthermore, the altered symmetry lead to the formation of periodically ordered, channellike vacancies within the superlattice. Hence, these materials could find possible applications in catalysis with discretely confined reaction sites. The channel like vacancies could be used to host guests within the superstructures like small conducting molecules or even a second nanoparticle species. Such guests could act as charge collector/carrier agents and further alter the properties of the nanoparticle-based material. On the road toward nanoparticle based materials, the relevance of periodic positioning and spatial orientation of nanoparticles was already analyzed by Smalyukh et al. for triclinic colloidal crystals consisting of upconversion nanoparticles. ${ }^{[69]}$ With the work presented here, we add the aspect of direct shape-symmetry relation, which could be relevant to other nanoparticle systems as well. An aspect, we believe is highly relevant, is what we can learn about defect formation and influence on functional properties in colloidal superstructures formed by nanoparticles.

\section{Experimental Section}

All chemicals were used without further purification. Dry solvents were purified according to standard methods.

Synthesis of Triangular $\mathrm{ZnO}$ Nanorods: A mixture of $35 \mathrm{~mL}$ of cyclohexane p.a., $1.5 \mathrm{~mL} \mathrm{H} \mathrm{H}_{2} \mathrm{O}$ dest. and $1.9 \mathrm{mmol}$ ligand were placed into a $250 \mathrm{~mL}$ two-neck flask at $40{ }^{\circ} \mathrm{C}$ oil-bath temperature and treated with an ultrasonic lance (Bandelin Sonopuls HD3100 with a MS73 microtip) at $65 \%$ intensity. After $20 \mathrm{~min}$ a solution of $1 \mathrm{~g}(1.6 \mathrm{mmol})$

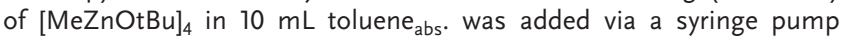
(KD Scientific KS200) at an addition rate of $0.7 \mathrm{~mL} \mathrm{~min}{ }^{-1}\left(0.1 \mathrm{mmol} \mathrm{min}^{-1}\right)$.

Synthesis of Hexagonal $\mathrm{ZnO}$ Nanorods: The synthesis of the here used hexagonal $\mathrm{ZnO}$ nanorods was reported earlier. ${ }^{[4]]}$

Nanoparticle Self-Assembly: Both hexagonal and triangular $\mathrm{ZnO}$ nanorods were assembled according to the method of Dong et al. [12] $3 \mathrm{~mL}$ of diethylene glycol (DEG) was spread into a Petri dish with $4 \mathrm{~cm}$ diameter. Afterwards, the DEG was overlaid with $200 \mu \mathrm{L}$ of $3.75 \mathrm{mg} \mathrm{mL}^{-1}$ nanoparticle dispersion in toluene. After the addition of the nanoparticle dispersion, the Petri dish was covered with a glass lid to ensure slow evaporation of the dispersion solvent. After $24 \mathrm{~h}$ the resulting nanoparticle sheets were collected by scooping them with a carbon coated copper TEM-grid or a silicon-wafer substrate. To remove residual DEG, the TEM-grids and silicon-wafer substrates were kept in a vacuum chamber for $5 \mathrm{~h}$.

Analysis of the Commercial Monoolein: The composition of the commercial monoolein was determined by means of direct inlet APCI (atmospheric pressure chemical ionization) mass spectrometry using a Varian MS 320 triple quadrupole mass analyzer. Samples dissolved in acetonitrile/water (95\%/5\%; both with $0.1 \%$ formic acid) were introduced into the $\mathrm{APCl}$ source using a syringe pump at a flow rate of

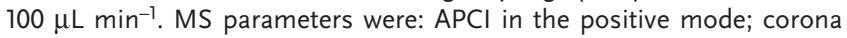
current: $2.0 \mu \mathrm{A}$; nebulizer gas pressure: $379 \mathrm{kPa}$; drying gas: $172 \mathrm{kPa}$ at $200{ }^{\circ} \mathrm{C}$; vaporizer gas $82.7 \mathrm{kPa}$ at $400{ }^{\circ} \mathrm{C}$, and detector voltage at $1200 \mathrm{~V}$. Resulting protonated substances were detected in a range of $\mathrm{m} / \mathrm{z} 330$ to $\mathrm{m} / \mathrm{z}$ 930. In each case the most intense ion $\left(\mathrm{MH}+-\mathrm{H}_{2} \mathrm{O}\right)$ was used for quantitation and calculation (in triplicate $\pm S D$ ) of the mass fraction of the respective olein. The commercial monoolein consisted of mono- and diolein in ratio of $42.0 / 58.0 \pm 1.5$. Other constituents than oleins could not be excluded and product purity not specified.

Ligand Synthesis: 2,3-Dihydroxypropyl (Z)-9-octadecenoat $(X)$ was adapted from Mori et al. ${ }^{[0]}$

2,2-Dimethyl-1,3-dioxolane-4-methyl (Z)-9-octadecenoate (A): (土)-2,2-Dimethyl-1,3-dioxolane-4-methanol (2,3-acetone glycerol, $2.34 \mathrm{~g}$, $17.7 \mathrm{mmol}$ ), (Z)-9-octadecenoic acid (oleic acid, $5.00 \mathrm{~g}, 17.7 \mathrm{mmol}$ ) and 4-(dimethylamino)-pyridine (DMAP, $0.13 \mathrm{~g}, 1.06 \mathrm{mmol}$ ) were dissolved in $90 \mathrm{~mL}$ dry DCM and cooled to $0^{\circ} \mathrm{C}$. A solution of $N, N^{\prime}$ dicyclohexylcarbodiimide (DCC, $7.30 \mathrm{~g}, 35.4 \mathrm{mmol}$ ) in $60 \mathrm{~mL}$ dry DCM was added dropwise and the solution stirred for $16 \mathrm{~h}$. The solution was diluted with $150 \mathrm{~mL} n$-hexane and precipitated dicyclohexylurea was removed by filtration over diatomaceous earth. After evaporation of solvent, the residue was purified via FC (petroleum ether/ ethyl acetate $15: 1)$ to yield $6.70 \mathrm{~g}(95 \%) \mathrm{A}$ as colorless oil. $R_{\mathrm{f}}(\mathrm{A})$ $=0.32$ (petroleum ether/ethyl acetate 10:1); ${ }^{1} \mathrm{H}-\mathrm{NMR}(400 \mathrm{MHz}$, $\left.\mathrm{CDCl}_{3}\right): \delta=5.41-5.27\left(\mathrm{~m}, 2 \mathrm{H}, \mathrm{CH}_{2} \mathrm{CHCHCH}_{2}\right), 4.34-4.26(\mathrm{~m}, 1 \mathrm{H}$, $\left.(\mathrm{C}=\mathrm{O}) \mathrm{OCH}_{2} \mathrm{CHCH}_{2} \mathrm{O}\right), 4.18-4.07\left(\mathrm{~m}, 2 \mathrm{H},(\mathrm{C}=0) \mathrm{OCH}_{2} \mathrm{CHCH}_{2} \mathrm{O}\right)$, 4.07-4.03 (m, $\left.1 \mathrm{H},(\mathrm{C}=\mathrm{O}) \mathrm{OCH}_{2} \mathrm{CHCH}_{2} \mathrm{O}\right), 3.72(\mathrm{dd}, J=8.4 \mathrm{~Hz}, 2.3 \mathrm{~Hz}$, $\left.1 \mathrm{H},(\mathrm{C}=\mathrm{O}) \mathrm{OCH}_{2} \mathrm{CHCH}_{2} \mathrm{O}\right), 2.32\left(\mathrm{t}, J=7.6 \mathrm{~Hz}, 2 \mathrm{H},(\mathrm{C}=\mathrm{O}) \mathrm{CH}_{2} \mathrm{CH}_{2}\right)$, 2.06-1.93 (m, $\left.4 \mathrm{H}, \mathrm{CH}_{2} \mathrm{CHCHCH}_{2}\right), 1.66-1.56\left(\mathrm{~m}, 2 \mathrm{H},(\mathrm{C}=\mathrm{O}) \mathrm{CH}_{2} \mathrm{CH}_{2}\right)$, $1.42\left(\mathrm{~s}, 3 \mathrm{H},\left(\mathrm{CH}_{3}\right)_{2} \mathrm{CO}_{2}\right), 1.36\left(\mathrm{~s}, 3 \mathrm{H},\left(\mathrm{CH}_{3}\right)_{2} \mathrm{CO}_{2}\right), 1.35-1.19\left(\mathrm{brm}, 2 \mathrm{OH}, \mathrm{CH}_{2}\right)$, $0.87\left(\mathrm{t}, J=6.9 \mathrm{~Hz}, 3 \mathrm{H}, \mathrm{CH}_{2} \mathrm{CH}_{3}\right) \mathrm{ppm} ;{ }^{13} \mathrm{C} \mathrm{NMR}\left(101 \mathrm{MHz}, \mathrm{CDCl}_{3}\right)$ : $\delta=173.7(\mathrm{C}=\mathrm{O}), 130.1,129.9\left(\mathrm{CH}_{2} \mathrm{CHCHCH}_{2}\right), 109.9\left(\left(\mathrm{CH}_{3}\right)_{2} \mathrm{CO}_{2}\right)$, $73.8 \quad\left((\mathrm{C}=\mathrm{O}) \mathrm{OCH}_{2} \mathrm{CHCH}_{2} \mathrm{O}\right), \quad 66.5 \quad\left((\mathrm{C}=\mathrm{O}) \mathrm{OCH}_{2} \mathrm{CHCH}_{2} \mathrm{O}\right), \quad 64.7$ $\left((\mathrm{C}=\mathrm{O}) \mathrm{OCH}_{2} \mathrm{CHCH}_{2} \mathrm{O}\right), 34.2\left((\mathrm{C}=\mathrm{O}) \mathrm{CH}_{2} \mathrm{CH}_{2}\right), 32.0,29.9,29.8,29.6$, 29.4, 29.3, $29.2\left(\mathrm{CH}_{2}\right), 27.4,27.3\left(\mathrm{CH}_{2} \mathrm{CHCHCH}_{2}\right), 25.0\left((\mathrm{C}=\mathrm{O}) \mathrm{CH}_{2} \mathrm{CH}_{2}\right)$, $22.8\left(\mathrm{CH}_{2} \mathrm{CH}_{3}\right), 14.2\left(\mathrm{CH}_{2} \mathrm{CH}_{3}\right) \mathrm{ppm}$

2,3-Dihydroxypropyl (Z)-9-octadecenoat (X): 2,2-Dimethyl-1,3-dioxolane4-methyl (Z)-9-octadecenoate (A) $(6.18 \mathrm{~g}, 15.6 \mathrm{mmol})$ was dissolved in $62 \mathrm{~mL}$ of $80 \%$ aqueous acetic acid and heated to $50^{\circ} \mathrm{C}$ for $2 \mathrm{~h}$. Solvent was removed in vacuo and the residue dissolved in diethyl ether and washed with saturated, aqueous $\mathrm{NaHCO}_{3}$. The organic phase was dried over $\mathrm{MgSO}_{4}$ and solvent removed to yield $5.4 \mathrm{~g}(97 \%)$ of $\mathrm{X}$ as white, waxy solid. $R_{\mathrm{f}}(\mathrm{X})=0.23$ (petroleum ether/ethyl acetate 1:1); ${ }^{1} \mathrm{H}$ NMR $(400 \mathrm{MHz}$, DMSO-d $\left.\mathrm{d}_{6}\right): \delta=5.38-5.27\left(\mathrm{~m}, 2 \mathrm{H}, \mathrm{CH}_{2} \mathrm{CHCHCH}_{2}\right), 4.83(\mathrm{~d}, J=5.3 \mathrm{~Hz}$, $1 \mathrm{H}, \mathrm{CH}(\mathrm{OH})), 4.59\left(\mathrm{t}, J=5.7 \mathrm{~Hz}, 1 \mathrm{H}, \mathrm{CH}_{2} \mathrm{OH}\right), 4.10-3.83(\mathrm{~m}, 2 \mathrm{H}$, $\left.(\mathrm{C}=\mathrm{O}) \mathrm{OCH}_{2} \mathrm{CH}\right), 3.66-3.58(\mathrm{~m}, 1 \mathrm{H}, \mathrm{CH}(\mathrm{OH})), 3.39-3.28\left(\mathrm{~m}, 2 \mathrm{H}, \mathrm{CH}_{2} \mathrm{OH}\right)$, 2.31-2.23 (m, $\left.2 \mathrm{H},(\mathrm{C}=\mathrm{O}) \mathrm{CH}_{2} \mathrm{CH}_{2}\right), 2.04-1.90\left(\mathrm{~m}, 4 \mathrm{H}, \mathrm{CH}_{2} \mathrm{CHCHCH}_{2}\right)$, 1.56-1.45 (m, $\left.2 \mathrm{H},(\mathrm{C}=\mathrm{O}) \mathrm{CH}_{2} \mathrm{CH}_{2}\right), 1.35-1.17$ (brm, $\left.20 \mathrm{H}, \mathrm{CH}_{2}\right), 0.85$ $\left(\mathrm{t}, J=6.9 \mathrm{~Hz}, 3 \mathrm{H}, \mathrm{CH}_{2} \mathrm{CH}_{3}\right) \mathrm{ppm} ;{ }^{13} \mathrm{C}$ NMR $\left(101 \mathrm{MHz}\right.$, DMSO-d $\mathrm{d}_{6}$ ): $\delta=172.8(\mathrm{C}=\mathrm{O}), 129.57,129.55\left(\mathrm{CH}_{2} \mathrm{CHCHCH}_{2}\right), 69.3(\mathrm{CH}(\mathrm{OH})), 65.5$ ( $\left.(\mathrm{C}=\mathrm{O}) \mathrm{OCH} \mathrm{CH}_{2}\right), 62.7\left(\mathrm{CH}_{2} \mathrm{OH}\right), 33.5\left((\mathrm{C}=\mathrm{O}) \mathrm{CH}_{2} \mathrm{CH}_{2}\right), 31.3,29.11,29.09,28.9$, 28.7, 28.61, 28.59, 28.57, 28.50, $28.48\left(\mathrm{CH}_{2}\right), 26.59,26.57\left(\mathrm{CH}_{2} \mathrm{CHCHCH}_{2}\right)$, $24.4\left((\mathrm{C}=\mathrm{O}) \mathrm{CH}_{2} \mathrm{CH}_{2}\right), 22.07\left(\mathrm{CH}_{2} \mathrm{CH}_{3}\right), 13.9\left(\mathrm{CH}_{2} \mathrm{CH}_{3}\right)$ ppm.

1,3 Di-(Z)-9-octadecenoyl 2-hxdroxypropane (Y) was adapted from Pérignon et al. ${ }^{[7]]}$ 
1,3-Di-(Z)-9-octadecenoyl-propane-2-one (B): 1,3-Dihydroxyacetone (2.00 g, $22.2 \mathrm{mmol}),(Z)-9-o c t a d e c e n o i c ~ a c i d ~(12.54 \mathrm{~g}, 44.4 \mathrm{mmol})$ and 4-(Dimethylamino)-pyridine $(5.43 \mathrm{~g}, 44.4 \mathrm{mmol})$ were dissolved in $50 \mathrm{~mL}$ dry DCM, a solution of $N, N^{\prime}$-dicyclohexylcarbodiimide $(9.16 \mathrm{~g}$, $44.4 \mathrm{mmol}$ ) in $25 \mathrm{~mL}$ dry DCM was added dropwise and the reaction mixture stirred for $24 \mathrm{~h}$. Precipitated dicyclohexylurea was removed by filtration over diatomaceous earth. After evaporation of solvent, the residue was purified via FC (petroleum ether/ethyl acetate 9:1 to 7.5:1) to yield $11.31 \mathrm{~g}(82 \%) \mathrm{B}$ as colorless solid. $R_{\mathrm{f}}(\mathrm{B})=0.68$ (petroleum ether/ethyl acetate 7:3); ${ }^{1} \mathrm{H}$ NMR $\left(500 \mathrm{MHz}^{\mathrm{C}} \mathrm{CDCl}_{3}\right): \delta=5.39-5.29$ (m, $\left.4 \mathrm{H}, \mathrm{CH}_{2} \mathrm{CHCHCH}_{2}\right), 4.74\left(\mathrm{~s}, 4 \mathrm{H}, \mathrm{OCH}_{2}(\mathrm{C}=\mathrm{O}) \mathrm{CH}_{2} \mathrm{O}\right), 2.41$ (t, $\left.J=7.6 \mathrm{~Hz}, \mathrm{O}(\mathrm{C}=\mathrm{O}) \mathrm{CH}_{2} \mathrm{CH}_{2}\right), 2.05-1.94\left(\mathrm{~m}, 8 \mathrm{H}, \mathrm{CH}_{2} \mathrm{CHCHCH}_{2}\right)$, 1.69-1.60 (m, $\left.4 \mathrm{H}, \mathrm{O}(\mathrm{C}=\mathrm{O}) \mathrm{CH}_{2} \mathrm{CH}_{2}\right), 1.39-1.20$ (brm, $\left.40 \mathrm{H}, \mathrm{CH}_{2}\right)$, $0.87\left(\mathrm{t}, 3 \mathrm{H}, \mathrm{CH}_{2} \mathrm{CH}_{3}\right)$ ppm; ${ }^{13} \mathrm{C}-\mathrm{NMR}\left(126 \mathrm{MHz}, \mathrm{CDCl}_{3}\right): \delta=$ $198.3\left(\mathrm{OCH}_{2}(\mathrm{C}=\mathrm{O}) \mathrm{CH}_{2} \mathrm{O}\right), \quad 173.0 \quad\left(\mathrm{O}(\mathrm{C}=\mathrm{O}) \mathrm{CH}_{2} \mathrm{CH}_{2}\right), \quad 130.2, \quad 129.9$ $\left(\mathrm{CH}_{2} \mathrm{CHCHCH}_{2}\right), 66.3\left(\mathrm{OCH}_{2}(\mathrm{C}=\mathrm{O}) \mathrm{CH}_{2} \mathrm{O}\right), 33.8\left(\mathrm{O}(\mathrm{C}=\mathrm{O}) \mathrm{CH}_{2} \mathrm{CH}_{2}\right)$, $32.0,29.9,29.8,29.6,29.4,29.3,29.19,29.16\left(\mathrm{CH}_{2}\right), 27.34,27.28$ $\left(\mathrm{CH}_{2} \mathrm{CHCHCH}_{2}\right), \quad 24.9 \quad\left(\mathrm{O}(\mathrm{C}=\mathrm{O}) \mathrm{CH}_{2} \mathrm{CH}_{2}\right), \quad 22.8 \quad\left(\mathrm{CH}_{2} \mathrm{CH}_{3}\right), \quad 14.2$ $\left(\mathrm{CH}_{2} \mathrm{CH}_{3}\right)$ ppm.

Di-(Z)-9-octadecenoyl 2-hxdroxypropane (Y): 1,3-Di-(Z)-9-octadecenoylpropane-2-one $(B, 11.31 \mathrm{~g}, 18.3 \mathrm{mmol}$ ) was dispersed in a mixture of $240 \mathrm{~mL} \mathrm{THF}$ and $16 \mathrm{~mL} \mathrm{H} \mathrm{H}_{2} \mathrm{O}$ and cooled to $0^{\circ} \mathrm{C} . \mathrm{NaBH}_{4}(1.59 \mathrm{~g}$, $42.0 \mathrm{mmol}$ ) was added in small portions and the reaction mixture stirred for $2 \mathrm{~h}$. The mixture was neutralized by dropwise addition of glacial acetic acid, resulting in the formation of a white precipitate. Solvent was evaporated and the residue dissolved in $200 \mathrm{~mL}$ DCM, washed with saturated, aqueous $\mathrm{NaHCO}_{3}$ and dried over $\mathrm{Na}_{2} \mathrm{SO}_{4}$. Solvent was evaporated and the crude product, also containing small amounts of 1,2-di-(Z)-9-octadecenoyl 3-hxdroxypropane, was purified via FC (petroleum ether/ethyl acetate 10:1 to 5:1), to yield $7.82 \mathrm{~g} \mathrm{Y}$ (69\%), $2.38 \mathrm{~g}(21 \%)$ of a 1:1 mixture of the region-isomers $Y$ and $Y 2$ and $0.23 \mathrm{~g}(2 \%)$ of pure 1,2-di-(Z)-9-octadecenoyl 3-hxdroxypropane (Y2) as colorless oils. $R_{f}(Y)=0.58$ (petroleum ether/ethyl acetate 7:3), $R_{f}(Y 2)=0.51$ (petroleum ether/ethyl acetate 7:3); ${ }^{1} \mathrm{H}-\mathrm{NMR}$ of Y $\left(500 \mathrm{MHz}, \mathrm{CDCl}_{3}\right): \delta=5.38-5.29\left(\mathrm{~m}, 4 \mathrm{H}, \mathrm{CH}_{2} \mathrm{CHCHCH}_{2}\right), 4.19-$ $4.09\left(\mathrm{~m}, 4 \mathrm{H}, \mathrm{OCH}_{2} \mathrm{CH}(\mathrm{OH}) \mathrm{CH}_{2} \mathrm{O}\right), 4.09-4.03\left(\mathrm{~m}, 1 \mathrm{H}, \mathrm{OCH}_{2} \mathrm{CH}(\mathrm{OH})\right.$ $\left.\mathrm{CH}_{2} \mathrm{O}\right), 2.60\left(\mathrm{~d}, 1 \mathrm{H}, \mathrm{OCH}_{2} \mathrm{CH}(\mathrm{OH}) \mathrm{CH}_{2} \mathrm{O}\right), 2.33$ (t, J $=7.5 \mathrm{~Hz}, 4 \mathrm{H}$, $\left.\mathrm{O}(\mathrm{C}=\mathrm{O}) \mathrm{CH}_{2} \mathrm{CH}_{2}\right), 2.04-1.94\left(\mathrm{~m}, 8 \mathrm{H}, \mathrm{CH}_{2} \mathrm{CHCHCH}_{2}\right), 1.66-1.56(\mathrm{~m}, 4 \mathrm{H}$ $\left.\mathrm{O}(\mathrm{C}=\mathrm{O}) \mathrm{CH}_{2} \mathrm{CH}_{2}\right), 1.37-1.19\left(\mathrm{brm}, 40 \mathrm{H}, \mathrm{CH}_{2}\right), 0.87(\mathrm{t}, J=7.1 \mathrm{~Hz}, 6 \mathrm{H}$, $\left.\mathrm{CH}_{2} \mathrm{CH}_{3}\right) \mathrm{ppm} ;{ }^{13} \mathrm{C}-\mathrm{NMR}$ of $\mathrm{Y}\left(126 \mathrm{MHz}, \mathrm{CDCl}_{3}\right): \delta=174.0(\mathrm{C}=\mathrm{O}), 130.1$, 129.8, $\left(\mathrm{CH}_{2} \mathrm{CHCHCH}_{2}\right), 68.4(\mathrm{CH}(\mathrm{OH})), 65.1\left(\mathrm{OCH}_{2} \mathrm{CH}(\mathrm{OH}) \mathrm{CH}_{2} \mathrm{O}\right)$, $34.2\left(\mathrm{O}(\mathrm{C}=\mathrm{O}) \mathrm{CH}_{2} \mathrm{CH}_{2}\right), 32.0,29.9,29.8,29.6,29.4,29.3,29.2\left(\mathrm{CH}_{2}\right)$, 27.32, $27.27\left(\mathrm{CH}_{2} \mathrm{CHCHCH}_{2}\right), 25.0\left(\mathrm{O}(\mathrm{C}=\mathrm{O}) \mathrm{CH}_{2} \mathrm{CH}_{2}\right), 22.8\left(\mathrm{CH}_{2} \mathrm{CH}_{3}\right)$, $14.2\left(\mathrm{CH}_{2} \mathrm{CH}_{3}\right)$ ppm; ${ }^{1} \mathrm{H}-\mathrm{NMR}$ of $\mathrm{Y} 2\left(500 \mathrm{MHz}, \mathrm{CDCl}_{3}\right): \delta=5.39-5.28$ (m, $\left.4 \mathrm{H}, \mathrm{CH}_{2} \mathrm{CHCHCH}_{2}\right), 5.10-5.04\left(\mathrm{~m}, 1 \mathrm{H},(\mathrm{C}=\mathrm{O}) \mathrm{OCH}_{2} \mathrm{CH}(\mathrm{O}(\mathrm{C}=\mathrm{O}))\right.$, 4.34-4.20 (m, $2 \mathrm{H},(\mathrm{C}=0) \mathrm{OCH}_{2} \mathrm{CH}(\mathrm{O}(\mathrm{C}=0)), 3.76-3.69(\mathrm{~m}, 2 \mathrm{H}$, $\left.\mathrm{CH}_{2} \mathrm{OH}\right), 2.37-2.28\left(\mathrm{~m}, 4 \mathrm{H}, \mathrm{O}(\mathrm{C}=\mathrm{O}) \mathrm{CH}_{2} \mathrm{CH}_{2}\right), 2.15$ (brm, $\left.1 \mathrm{H}, \mathrm{CH}_{2} \mathrm{OH}\right)$ 2.07-1.94 (m, 8 H, CH $\left.\mathrm{CHCHCH}_{2}\right), 1.66-1.57\left(\mathrm{~m}, 4 \mathrm{H}, \mathrm{O}(\mathrm{C}=\mathrm{O}) \mathrm{CH}_{2} \mathrm{CH}_{2}\right)$, 1.39-1.19 (brm, $40 \mathrm{H}, \mathrm{CH}_{2}$ ), 0.87 (t, J = 7.1 Hz, $\left.6 \mathrm{H}, \mathrm{CH}_{2} \mathrm{CH}_{3}\right)$ ppm; ${ }^{13} \mathrm{C}$ NMR of $\mathrm{Y} 2\left(126 \mathrm{MHz}, \mathrm{CDCl}_{3}\right): \delta=173.9\left((\mathrm{C}=\mathrm{O}) \mathrm{OCH}_{2} \mathrm{CH}(\mathrm{O}(\mathrm{C}=\mathrm{O}))\right.$, $173.6\left((\mathrm{C}=\mathrm{O}) \mathrm{OCH}_{2} \mathrm{CH}(\mathrm{O}(\mathrm{C}=\mathrm{O})), 130.2,129.8\left(\mathrm{CH}_{2} \mathrm{CHCHCH}_{2}\right), 72.3\right.$ $\left((\mathrm{C}=\mathrm{O}) \mathrm{OCH}_{2} \mathrm{CH}(\mathrm{O}(\mathrm{C}=\mathrm{O})), \quad 62.2 \quad\left(\left((\mathrm{C}=\mathrm{O}) \mathrm{OCH}_{2} \mathrm{CH}(\mathrm{O}(\mathrm{C}=\mathrm{O})), \quad 61.7\right.\right.\right.$ $\left(\mathrm{CH}_{2} \mathrm{OH}\right), 34.4,34.2\left(\mathrm{O}(\mathrm{C}=\mathrm{O}) \mathrm{CH}_{2} \mathrm{CH}_{2}\right), 32.0,29.9,29.8,29.7,29.5$, 29.3, 29.24, 29.22, $29.19\left(\mathrm{CH}_{2}\right), 27.4,27.3\left(\mathrm{CH}_{2} \mathrm{CHCHCH}_{2}\right), 25.05,25.00$ $\left(\mathrm{O}(\mathrm{C}=\mathrm{O}) \mathrm{CH}_{2} \mathrm{CH}_{2}\right), 22.8\left(\mathrm{CH}_{2} \mathrm{CH}_{3}\right), 14.2\left(\mathrm{CH}_{2} \mathrm{CH}_{3}\right)$ ppm.

1,2,3 Tri-(Z)-9-octadecenoyl propane (T): Commercial tri-Ol (Triolein, $80 \%$ ) was purchased from $A B C R$ and further purified via a Büchi Reveleris $\mathrm{X} 2$ medium pressure liquid chromatography (MPLC). ${ }^{7} \mathrm{H} N M R$ $\left(500 \mathrm{MHz}, \mathrm{CDCl}_{3}\right): \delta=5.42-5.28\left(\mathrm{~m}, 6 \mathrm{H}, \mathrm{CH}_{2} \mathrm{CHCHCH}_{2}\right), 5.28-5.22(\mathrm{~m}$, $\left.1 \mathrm{H}, \mathrm{OCH}_{2} \mathrm{CH}(\mathrm{O}) \mathrm{CH}_{2} \mathrm{O}\right), 4.31-4.10\left(\mathrm{~m}, 4 \mathrm{H}, \mathrm{OCH}_{2} \mathrm{CH}(\mathrm{O}) \mathrm{CH}_{2} \mathrm{O}\right), 2.33-$ $2.26\left(\mathrm{~m}, 6 \mathrm{H}, \mathrm{O}(\mathrm{C}=\mathrm{O}) \mathrm{CH}_{2} \mathrm{CH}_{2}\right), 2.07-1.92\left(\mathrm{~m}, 12 \mathrm{H}, \mathrm{CH}_{2} \mathrm{CHCHCH}_{2}\right)$, 1.65-1.54 (m, $\left.6 \mathrm{H}, \mathrm{O}(\mathrm{C}=\mathrm{O}) \mathrm{CH}_{2} \mathrm{CH}_{2}\right), 1.37-1.20\left(\mathrm{~m}, 60 \mathrm{H}, \mathrm{CH}_{2}\right), 0.87$ $\left(\mathrm{t}, J=7.1 \mathrm{~Hz}, 9 \mathrm{H}, \mathrm{CH}_{2} \mathrm{CH}_{3}\right) \mathrm{ppm} ;{ }^{13} \mathrm{C}-\mathrm{NMR}\left(126 \mathrm{MHz}, \mathrm{CDCl}_{3}\right): \delta=$ $173.3\left(\mathrm{CH}_{2} \mathrm{O}(\mathrm{C}=\mathrm{O})\right), 172.9\left(\mathrm{CHO}(\mathrm{C}=\mathrm{O}), 130.1,129.8\left(\mathrm{CH}_{2} \mathrm{CHCHCH}_{2}\right)\right.$, $69.0 \quad\left(\mathrm{OCH}_{2} \mathrm{CH}(\mathrm{O}) \mathrm{CH}_{2} \mathrm{O}\right), \quad 62.2 \quad\left(\mathrm{OCH}_{2} \mathrm{CH}(\mathrm{O}) \mathrm{CH}_{2} \mathrm{O}\right), \quad 34.3$ $\left(\mathrm{CH}(\mathrm{O})(\mathrm{C}=\mathrm{O}) \mathrm{CH}_{2}\right), 34.1\left(\mathrm{CH}_{2} \mathrm{O}(\mathrm{C}=\mathrm{O}) \mathrm{CH}_{2} \mathrm{CH}_{2}\right), 32.0,29.9-29.1\left(\mathrm{CH}_{2}\right)$, 27.32, $27.27\left(\mathrm{CH}_{2} \mathrm{CHCHCH}_{2}\right), 24.98,24.94\left(\mathrm{O}(\mathrm{C}=\mathrm{O}) \mathrm{CH}_{2} \mathrm{CH}_{2}\right), 22.8$ $\left(\mathrm{CH}_{2} \mathrm{CH}_{3}\right), 14.2\left(\mathrm{CH}_{2} \mathrm{CH}_{3}\right)$ ppm.
Analytical Methods: FT-IR spectra were obtained with a Perkin-Elmer Spectrum 100. TEM micrographs were taken on a Zeiss Libra120 with $120 \mathrm{kV}$ acceleration voltage. HR-TEM images were acquired with a JEOL, JEM 2200FS at an accelerating voltage of $200 \mathrm{kV}$. SEM images were acquired with a Zeiss Gemini 500. Powder X-ray diffraction was performed with a Bruker D8 Discover diffractometer using Cu-Ko radiation. UV-Vis spectra have been acquired using a Varian Cary 100 Scan. DLS measurements have been performed with a Malvern Zetasizer Nano ZSP. NMR spectra were measured on a Varian Unity INOVA 400 spectrometer. NMR spectra were recorded on a JEOL $500(500 \mathrm{MHz})$ and on a Bruker Avance III 400 (400 MHz). Chemical shifts are reported using solvent signals as internal reference. Two-dimensional spectra (COSY, HSQC, HMBC) of ${ }^{1} \mathrm{H}-{ }^{1} \mathrm{H}$ and ${ }^{1} \mathrm{H}-{ }^{13} \mathrm{C}$ were utilized for specific signal assignment.

\section{Supporting Information}

Supporting Information is available from the Wiley Online Library or from the author.

\section{Acknowledgements}

The research was funded by the German Research Foundation (DFG) within the collaborative research center SFB-1214 project A1 and project Z1 Particle Analysis Center. The spelling error in the author's name (V.W.) was corrected on February 17, 2021 after initial online publication.

Open access funding enabled and organized by Projekt DEAL.

\section{Conflict of Interest}

The authors declare no conflict of interest.

\section{Keywords}

colloidal superstructures, nanoparticle shape, nanorods, particle-based materials, self-assembly

Received: October 25, 2020

Revised: November 23, 2020

Published online: December 18, 2020

[1] A. P. Alivisatos, Science 1996, 271, 933.

[2] X. Peng, L. Manna, W. Yang, J. Wickham, E. Scher, A. Kadavanich, A. P. Alivisatos, Nature 2000, 404, 59.

[3] A. P. Alivisatos, J. Phys. Chem. 1996, 100, 13226.

[4] C. B. Murray, D. J. Norris, M. G. Bawendi, J. Am. Chem. Soc. 1993, 115,8706

[5] S. L. Cumberland, K. M. Hanif, A. Javier, G. A. Khitrov, G. F. Strouse, S. M. Woessner, C. S. Yun, Chem. Mater. 2002, 14, 1576.

[6] C. B. Murray, C. R. Kagan, M. G. Bawendi, Science 1995, 270, 1335.

[7] D. V. Talapin, E. V. Shevchenko, M. I. Bodnarchuk, X. Ye, J. Chen, C. B. Murray, Nature 2009, 461, 964.

[8] L. Schertel, I. Wimmer, P. Besirske, C. M. Aegerter, G. Maret, S. Polarz, G. J. Aubry, Phys. Rev. Mater. 2019, 3, 015203.

[9] D. Zámbó, A. Schlosser, P. Rusch, F. Lübkemann, J. Koch, H. Pfnür, N. C. Bigall, Small 2020, 16, 1906934.

[10] S. Naskar, A. Freytag, J. Deutsch, N. Wendt, P. Behrens, A. Köckritz, N. C. Bigall, Chem. Mater. 2017, 29, 9208.

[11] N. C. Bigall, A.-K. Herrmann, M. Vogel, M. Rose, P. Simon, W. Carrillo-Cabrera, D. Dorfs, S. Kaskel, N. Gaponik, A. Eychmüller, Angew. Chem., Int. Ed. 2009, 48, 9731. 
[12] A. Dong, J. Chen, P. M. Vora, J. M. Kikkawa, C. B. Murray, Nature 2010, 466, 474.

[13] X. Ye, L. Jin, H. Caglayan, J. Chen, G. Xing, C. Zheng, V. Doan-Nguyen, Y. Kang, N. Engheta, C. R. Kagan, C. B. Murray, ACS Nano 2012, 6, 2804.

[14] K. Miszta, J. de Graaf, G. Bertoni, D. Dorfs, R. Brescia, S. Marras, L. Ceseracciu, R. Cingolani, R. van Roij, M. Dijkstra, L. Manna, Nat. Mater. 2011, 10, 872.

[15] G. Singh, H. Chan, A. Baskin, E. Gelman, N. Repnin, P. Král, R. Klajn, Science 2014, 345, 1149 .

[16] T. Wang, J. Zhuang, J. Lynch, O. Chen, Z. Wang, X. Wang, D. LaMontagne, H. Wu, Z. Wang, Y. C. Cao, Science 2012, 338, 358.

[17] R.-Q. Song, H. Cölfen, Adv. Mater. 2010, 22, 1301.

[18] S. Polarz, Adv. Funct. Mater. 2011, 21, 3214.

[19] M. Calvaresi, Nat. Nanotechnol. 2020, 15, 512.

[20] L. Boselli, H. Lopez, W. Zhang, Q. Cai, V. A. Giannone, J. Li, A. Moura, J. M. de Araujo, J. Cookman, V. Castagnola, Y. Yan, K. A. Dawson, Commun. Mater. 2020, 1, 35.

[21] L. Manna, D. J. Milliron, A. Meisel, E. C. Scher, A. P. Alivisatos, Nat. Mater. 2003, 2, 382.

[22] L. Manna, E. C. Scher, L.-S. Li, A. P. Alivisatos, J. Am. Chem. Soc. 2002, 124, 7136

[23] A. E. Saunders, A. Ghezelbash, P. Sood, B. A. Korgel, Langmuir 2008, 24, 9043

[24] I. Chakraborty, N. Feliu, S. Roy, K. Dawson, W. J. Parak, Bioconjugate Chem. 2018, 29, 1261.

[25] M. Chen, T. Pica, Y.-B. Jiang, P. Li, K. Yano, J. P. Liu, A. K. Datye, H. Fan, J. Am. Chem. Soc. 2007, 129, 6348.

[26] D. Wang, Y. Kang, X. Ye, C. B. Murray, Chem. Mater. 2014, 26, 6328.

[27] H. Zhong, S. S. Lo, T. Mirkovic, Y. Li, Y. Ding, Y. Li, G. D. Scholes, ACS Nano 2010, 4, 5253

[28] X.-S. Du, M. Mo, R. Zheng, S.-H. Lim, Y. Meng, Y.-W. Mai, Cryst. Growth Des. 2008, 8, 2032

[29] F. Dumestre, B. Chaudret, C. Amiens, M. Respaud, P. Fejes, P. Renaud, P. Zurcher, Angew. Chem., Int. Ed. 2003, 42, 5213.

[30] X. Huang, B. Li, C. Peng, G. Song, Y. Peng, Z. Xiao, X. Liu, J. Yang, L. Yu, J. Hu, Nanoscale 2016, 8, 1040.

[31] Z. Zhang, X. Zhong, S. Liu, D. Li, M. Han, Angew. Chem., Int. Ed. 2005, 44, 3466.

[32] L. Hou, Q. Zhang, L. Ling, C.-X. Li, L. Chen, S. Chen, J. Am. Chem. Soc. 2013, 135, 10618

[33] C. B. Ong, L. Y. Ng, A. W. Mohammad, Renewable Sustainable Energy Rev. 2018, 81, 536.

[34] Ü. Özgür, Y. I. Alivov, C. Liu, A. Teke, M. A. Reshchikov, S. Doğan, V. Avrutin, S. J. Cho, H. Morkoç, J. Appl. Phys. 2005, 98, 041301.

[35] Z. L. Wang, J. Phys.: Condens. Matter 2004, 16, R829.

[36] M. Ramzan Parra, P. Pandey, H. Siddiqui, V. Sudhakar, K. Krishnamoorthy, F. Z. Haque, Appl. Surf. Sci. 2019, 470, 1130.

[37] H. W. Noh, S. M. Jeong, J. Cho, J.-I. Hong, Nanoscale 2018, 10, 6801.

[38] Y. Liu, H. Liu, Q. Zhang, T. Li, RSC Adv. 2017, 7, 3515.

[39] E. Kowsari, S. Abdpour, J. Solid State Chem. 2017, 256, 141.

[40] A. Akbari, A. A. Firooz, J. Beheshtian, A. A. Khodadadi, Appl. Surf. Sci. 2014, 315, 8 .
[41] S. Theiss, M. Voggel, M. Schlötter, S. Sutter, M. T. Stöckl, S. Polarz, CrystEngComm 2019, 21, 5137.

[42] M. H. Xie, S. M. Seutter, W. K. Zhu, L. X. Zheng, H. Wu, S. Y. Tong, Phys. Rev. Lett. 1999, 82, 2749.

[43] N. Pinna, K. Weiss, J. Urban, M. P. Pileni, Adv. Mater. 2001, 13, 261.

[44] T. Andelman, Y. Gong, M. Polking, M. Yin, I. Kuskovsky, G. Neumark, S. O'Brien, J. Phys. Chem. B 2005, 109, 14314.

[45] J. H. Warner, R. D. Tilley, Adv. Mater. 2005, 17, 2997.

[46] X. Wang, J. Song, Z. L. Wang, Chem. Phys. Lett. 2006, 424, 86.

[47] M. H. Xie, M. Gong, E. K. Y. Pang, H. S. Wu, S. Y. Tong, Phys. Rev. B 2006, 74, 085314

[48] D. S. Wang, T. Xie, Q. Peng, S. Y. Zhang, J. Chen, Y. D. Li, Chem. - Eur. J. 2008, 14, 2507.

[49] G. Muñoz-Hernández, A. Escobedo-Morales, U. Pal, Cryst. Growth Des. 2009, 9, 297.

[50] U. Pal, C. W. Kim, N. A. Jadhav, Y. S. Kang, J. Phys. Chem. C 2009, $113,14676$.

[51] X. Chen, F. Fang, A. M. C. Ng, A. B. Djurisic, S. Tong, J. Electrochem. Soc. 2010, 157, K269.

[52] W. S. Chiu, A. Yaghoubi, M. Y. Chia, N. H. Khanis, S. A. Rahman, P. S. Khiew, Y.-L. Chueh, CrystEngComm 2014, 16, 6003.

[53] S. Ghosh, R. Gaspari, G. Bertoni, M. C. Spadaro, M. Prato, S. Turner, A. Cavalli, L. Manna, R. Brescia, ACS Nano 2015, 9, 8537.

[54] X. Yin, Y. Shi, Y. Wei, Y. Joo, P. Gopalan, I. Szlufarska, X. Wang, Langmuir 2017, 33, 7708.

[55] C. Lizandara-Pueyo, S. Siroky, M. R. Wagner, A. Hoffmann, J. S. Reparaz, M. Lehmann, S. Polarz, Adv. Funct. Mater. 2011, 21, 295.

[56] M. Gerigk, J. Bahner, T. Kollek, S. Helfrich, R. Rosenberg, H. Cölfen, S. Polarz, Part. Part. Syst. Charact. 2017, 34, 1600215.

[57] M. Gerigk, P. Ehrenreich, M. R. Wagner, I. Wimmer, J. S. Reparaz, C. M. Sotomayor Torres, L. Schmidt-Mende, S. Polarz, Nanoscale 2015, 7, 16969.

[58] G. te Velde, F. M. Bickelhaupt, E. J. Baerends, C. F. Guerra, S. J. A. van Gisbergen, J. G. Snijders, T. Ziegler, J. Comput. Chem. 2001, 22, 931.

[59] J. P. Perdew, K. Burke, M. Ernzerhof, Phys. Rev. Lett. 1996, 77, 3865.

[60] J. A. Todd, Math. Gaz. 1950, 34, 239

[61] M. R. Querry, Optical constants, Contractor Report CRDC-CR-85034, 1985.

[62] A. V. Kildishev, A. Boltasseva, V. M. Shalaev, Science 2013, 339, 1232009.

[63] E. Maguid, I. Yulevich, D. Veksler, V. Kleiner, M. L. Brongersma, E. Hasman, Science 2016, 352, 1202.

[64] M. Khorasaninejad, F. Capasso, Science 2017, 358, eaam8100.

[65] J.-M. Nam, J.-W. Oh, H. Lee, Y. D. Suh, Acc. Chem. Res. 2016, 49, 2746.

[66] J.-F. Masson, ACS Sens. 2017, 2, 16.

[67] A. Ryabov, J. W. Thurner, D. Nabben, M. V. Tsarev, P. Baum, Sci. Adv. 2020, 6, eabbl393.

[68] K. J. Mohler, D. Ehberger, I. Gronwald, C. Lange, R. Huber, P. Baum, Sci. Adv. 2020, 6, eabc8804.

[69] H. Mundoor, B. Senyuk, I. I. Smalyukh, Science 2016, 352, 69.

[70] K. Mori, Tetrahedron 2012, 68, 8441.

[71] M. Pérignon, J. Lecomte, M. Pina, A. Renault, C. Simonneau-Deve, P. Villeneuve, J. Am. Oil Chem. Soc. 2012, 89, 89. 